\title{
On Correlation and Default Clustering in Credit Markets*
}

\author{
Antje Berndt ${ }^{\dagger}$ \\ Peter Ritchken ${ }^{\ddagger}$ \\ Zhiqiang $\operatorname{Sun}^{\S}$
}

October 25, 2009

\begin{abstract}
We establish Markovian models in the Heath, Jarrow and Morton paradigm that permit an exponential affine representation of riskless and risky bond prices while offering significant flexibility in the choice of volatility structures. Estimating models in our family is typically no more difficult than estimating term structure models in the workhorse affine family. In addition to diffusive and jump-induced default correlations, default events can impact credit spreads of surviving firms. This feature allows a greater clustering of defaults. Numerical implementations highlight the importance of taking interest rate-credit spread correlations, credit spread impact factors and the full credit spread curve information into account when building a unified model framework that prices any credit derivative.
\end{abstract}

JEL Classifications: C32; C51; G12

Keywords: Markovian HJM Models; Credit Derivatives; Default Clustering; Counterparty Credit Risk

\footnotetext{
${ }^{*}$ We are grateful to two anonymous referees for excellent comments and suggestions. We would like to thank Pierre Collin-Dufresne, David Lando, Dmitry Kramkov, Steve Shreve and Raman Uppal for extended discussions and comments.

${ }^{\dagger}$ Tepper School of Business, Carnegie Mellon University, Pittsburgh, PA 15213; aberndt@andrew.cmu.edu

${ }^{\ddagger}$ Weatherhead School of Management, Case Western Reserve University, Cleveland, OH 44106; phr@po.cwru.edu

$\S$ Weatherhead School of Management, Case Western Reserve University, Cleveland, OH 44106; zxs9@po.cwru.edu
} 
In light of the recent financial crisis, credit risk models, and the way they are used, have become the target of much criticism. ${ }^{1}$ A common concern is that the basic statistical properties of market data are not the same in a crisis as they are during stable periods (Danielsson (2002)). Hence the same models that price sophisticated financial instruments during normal times are virtually useless during times of crisis. The insight that statistical models fail at times when credit markets need them the most may motivate the search for a general framework in which stress testing can be easily conducted.

In response to previous market downturns, the Basel Committee on Banking Supervision already requires banks to conduct stress tests to limit their risk exposure throughout the business cycle (Basel II (2006)). That notion was recently reinforced when the Federal Reserve urged major investment banks to stress test their balance sheets in order to make sure they could survive under the sort of pressures that led to the near-collapse of Bear Stearns (Quinn (2008)). But according to a recent survey conducted by the Economist Intelligence Unit of The Economist (2008), 85 percent of senior executives of financial services firms worldwide say that their stress-testing practices are not adequate and need to be improved.

This paper develops a Markovian framework in the Heath, Jarrow, and Morton (1992) (hereafter HJM) paradigm for pricing credit derivatives on both single and multiple names. It is uniquely suitable for credit risk stress testing since it is both tractable and general at the same time. In particular, the models we develop have the following properties. First, our framework, being Markovian, permits the riskless and risky credit spread curves to be analytically computed at any point in time, based on a limited collection of state variables. Second, the models fully incorporate the current riskless term structure information as well as the full credit spread curve information for each firm. Third, the term structure of interest rate and credit spread volatilities could be time homogeneous, level dependent, and can be initialized to closely match the observable term structures of volatilities. Fourth, the actual structure for the volatilities of instantaneous interest rates and credit spreads can be selected from a large class, and need not be restricted to the affine family of Duffie and Kan (1996). Fifth, the models allow for arbitrary correlations between riskless interest rates and credit spreads as well as arbitrary correlations between credit spreads of different firms. Sixth, the models permit shocks to the economy that cause interest rates to jump as well as credit spreads of firms to change. Finally, we permit the default of some firms to cause jumps in the term structure of credit spreads of other surviving firms, a feature that allows defaults to cluster over time.

Duffie and Singleton (1999b), Schönbucher (2000), and others, previously showed how the HJM paradigm can be extended to include risky debt. Specifically, necessary restrictions on the dynamics of drift terms of forward rates and risky forward credit spreads have been identified that permit risky bonds to be priced in an arbitrage-free environment. Unfortunately, the resulting dynamics of all riskless forward rates and risky forward credit spreads are not in general Markov in a finite number of state variables. As a result, implementing these existing models, even via

\footnotetext{
${ }^{1}$ For recent commentary see, for example, Larsen (2007), Whitehouse (2007) and Danielsson (2008).
} 
Monte Carlo simulation, is delicate and computationally intensive. The problem is compounded further if the derivative security that needs to be priced depends on the credit spreads of multiple names. In contrast, by imposing modest restrictions on forward volatilities and jumps we are able to obtain low-dimensional Markovian representations for the term structures of riskless rates and credit spreads. The constraints will leave free the specification of the volatilities of instantaneous riskless rates and credit spreads for each name, and only involve curtailing the volatilities of forward rates relative to their spot rates.

Most studies of dynamic term structure models have focused on cases where both the drift vector and the instantaneous covariance matrix are affine functions of the state variables. Duffie and Kan (1996) establish that these affine models allow for an exponential affine association between bond prices and the underlying state variables. This appealing property has made affine models the workhorse term structure models over the past decade. ${ }^{2}$ However, the analytical tractability of affine term structure models comes at a cost. Specifically, the admissibility conditions entail a tradeoff where reasonable degrees of stochastic or level-dependent volatility can only be obtained at the expense of restricting correlations to perhaps unreasonable levels. Indeed, Dai and Singleton (2003) study this tradeoff and conclude that to obtain negative correlations between state variables, a feature particularly important if the state variables include the spot riskless rate and spot default intensity, the degree of heteroscedasticity in yields and/or spreads has to be dramatically curtailed.

Ahn, Dittmar, and Gallant (2002) extend affine to quadratic term structure models and show that the latter can permit flexibility in correlations while having a broader choice of yield heteroscedasticity. Using risk-free term structure data, Brandt and Chapman (2002) conclude that quadratic term structure models are superior to affine models in terms of fitting historical volatility. But quadratic models also have some drawbacks, in that, unlike affine models, they are not that easy to estimate and they cannot readily handle regime shifts and jumps in the state variables. For the risky term structure, Berndt (2007) employs nonparametric specification tests developed in Hong and $\mathrm{Li}$ (2005) to evaluate one-factor reduced-form credit risk models. She shows that popular univariate affine model specifications are strongly rejected by the data, findings which also are consistent with the evidence in Pan and Singleton (2008). Given the limitations of the workhorse term structure models, it seems desirable to search for a larger class of specifications that complements the affine class of Duffie-Kan, while at the same time retaining analytical tractability.

In this paper we establish families of models which permit analytical representations of riskless and risky bond prices. Perhaps somewhat surprisingly, our analytical representations of the yield curve and credit spread curves are affine functions of the state variables, even though our models are typically outside the Duffie-Kan framework. We accomplish this by curtailing volatility structures of forward rates and forward credit spreads relative to their spot rate counterparts without unnecessarily curtailing the spot rate volatilities. The number of state variables that result depends largely on the type of constraint imposed, with the most restrictive constraint leading to

\footnotetext{
${ }^{2}$ Dai and Singleton (2000) specify restrictions that produce a maximally flexible and empirically identifiable affine term structure model.
} 
the smallest collection of state variables. For example, in a model where riskless rates are driven by one factor and credit spreads by a correlated second factor, the restriction requires forward rate volatilities relative to their spot rate volatilities to be decaying exponentially as a function of their maturities. A less restrictive model, however, could permit volatility humps in both the term structures of riskless volatilities as well as the term structure of credit spread volatilities, properties that have been well documented in the literature and properties that cannot be accommodated in affine models of the same dimensionality (Dai and Singleton (2003)).

The advantage of our results is that we now have a large class of arbitrage-free riskless and credit spread term structure models to work with that allows us to entertain models with level dependent volatilities that permit positive as well as negative correlations between interest rates and credit spreads. Equally exciting, the family of models we establish can be estimated in similar ways to the affine models. Indeed, the implementation issues are typically less difficult since the more interesting affine models often require numerical solutions to systems of Riccati differential equations (Duffie, Pan, and Singleton (2000)). Such requirements are absent when estimating our models.

When the credit spread dynamics and jumps are shut down, our models reduce to the multivariate extensions of Ritchken and Sankarasubramanian (1995), developed by Inui and Kijima (1998). ${ }^{3}$ With credit spreads, our HJM models become more interesting, especially if credit spreads are correlated with interest rates, and if jumps are permitted. For such cases, we identify volatility restrictions that ensure that finite-state-variable models can be identified which make implementation issues associated with the HJM paradigm easy to address. ${ }^{4}$

Using time series data on term structures of both riskless rates and credit spreads, we illustrate how models within this family can be estimated using extended Kalman filters. We also provide a detailed road map for future empirical studies and show that, from a practical perspective, estimating models in our family is typically no more difficult than estimating models in the workhorse affine family of Duffie-Kan. We also provide two applications to illustrate the important role of correlations between interest rates and credit spreads. First, we investigate options on risky debt. Second, we value insurance contracts that offer protection against default of a counterparty to an underlying derivatives position. For both applications we find prices to be very sensitive to the interest rate-credit spread correlations.

After establishing powerful theory for single-name credit derivatives, we extend our framework to allow stress tests to be conducted for credit contracts that depend on multiple names. In our models, we introduce default correlation in three ways. First, we correlate the intensity processes

\footnotetext{
${ }^{3}$ For further Markovian models of the riskless term structure, see Ramaprasad and Chiarella (1995).

${ }^{4}$ Specifying a HJM model actually requires specifying structures for the volatilities of forward rates, and a family of forward rate curves, such as the Nelson-Siegel family, under which the forward rate curve is initialized. The specific model and family of curves for the calibration are said to be consistent if all forward rates produced by the model are contained in the family of forward rate curves used in calibration. A series of interesting papers have addressed this consistency issue, including Bjork and Christensen (1999), Bjork and Svensson (2001), La Chioma and Piccoli (2007) and the references therein.
} 
of different firms. Second, we allow shocks to the economy to cause jumps not only to the riskless yield curve but also to the credit spread curves of individual firms. Third, we permit the default of certain firms to affect the entire credit spread curve of other surviving firm, with the size of the impact depending on the surviving firm, the firm that defaulted and on the maturity of the forward credit spread. In this regard, our models are an extension of Jarrow and Yu (2001), and especially $\mathrm{Yu}(2007) .^{5}$

With these additions, clustering of defaults is permissible. Further, unlike most copula models, where the risk-neutral probabilities of default are static, our models permit risk-neutral default probabilities to adjust dynamically in response to changing information. ${ }^{6}$ We provide two illustrative applications of the resulting models. The first extends our earlier example of pricing an insurance contract that protects against counterparty credit risk in an underlying derivatives contract to the case where the insurer itself may not be immune from default. Our numerical results highlight the necessity of including contagion effects in our models. The second application demonstrates that our family of models can be implemented for a large portfolio of names. Specifically, we price CDS index tranches, where the underlying pool consists of a portfolio of 100 firms, each credit spread curve of which is modeled by its own dynamics and appropriately initialized to its date- 0 values. Interestingly, since information is used on all credits in the portfolio, we can easily explore how heterogeneity in the composition of the portfolio affect tranche values.

Our models permit events to occur which can trigger dramatic interest and/or credit shocks that may permeate through a large number of firms. They are therefore suited for stress testing alternative systemic scenarios. In light of the recent financial crisis, the proposed models offer a unique framework to study the impact of small probability events that cause large correlated losses throughout the economy.

The paper proceeds as follows. In Section 1, we describe general HJM models of riskless and risky term structures. In Section 2, we present our main results that allow us to price derivatives contracts on single names using Markovian HJM models. Section 3 illustrates how model parameters can be estimated using extended Kalman filtering, and highlights the importance of interest ratecredit spread correlations using two numerical examples. In Section 4, we extend the analysis to portfolios of credits where default of certain firms can impact the credit spread curves of other firms, provide applications and perform a number of stress tests. Section 5 concludes.

\section{HJM Models for Defaultable Bonds}

Consider a collection of $I$ firms. The default state of these firms is summarized by the process $Y(t)=\left(Y_{1}(t), Y_{2}(t), \ldots, Y_{I}(t)\right)$, where $Y_{i}(t)=1$ if firm $i$ has defaulted by time $t$, and $Y_{i}(t)=0$ otherwise. Set $\tau_{i}=\inf \left\{t \mid Y_{i}(t)=1\right\}$, and let $X(t)$ be a vector of state variables that influence

\footnotetext{
${ }^{5}$ This incorporates findings by Collin-Dufresne, Goldstein, and Helwege (2003) and Jorion and Zhang (2007) that major credit events at one firm can be associated with significant increases in spreads of other firms.

${ }^{6} \mathrm{Li}$ (2000) describes the copula approach to modeling default correlation.
} 
riskless yields and credit spreads of corporate debt. Given the state variables $(X(t), Y(t))$, the defaults of surviving firms over the next time increment are independent, time-inhomogeneous Poisson events. The risk-neutral default intensity for firm $i$ at date $t$ is $\eta_{i}(t)$. In addition to the default intensity for each firm, we also require assumptions on recovery given default. Let $\ell_{i}(t)$ denote the risk-neutral expected fractional loss in market value if default of firm $i$ were to occur at time $t$, conditional on the information available up to time $t$. Both $\eta_{i}(t)$ and $\ell_{i}(t)$ could depend on the state variables $(X(t), Y(t))$. The instantaneous credit spread, $\lambda_{i}(t)$, relates to the default intensity $\eta_{i}(t)$ by $\lambda_{i}(t)=\eta_{i}(t) \ell_{i}(t)$. The modeling of the credit spreads can be delicate because it is here where interesting dependence between defaults arises, and care has to be taken to ensure that feedback effects between $Y(t)$ and $X(t)$ are properly taken into account.

We begin our analysis by first focusing on an important element of $X(t)$, namely the riskless term structure that certainly affects the yields on corporate debt. Let $P(t, T)$ be the price at date $t$ of a pure riskless discount bond that pays $\$ 1$ at date $T$. Then:

$$
P(t, T)=e^{-\int_{t}^{T} f(t, u) d u},
$$

where $f(t, u)$ represents the date- $t$ forward rate for the future time increment $[u, u+d t]$. We assume that the risk-neutral dynamics of forward riskless rates follow a jump-diffusion process of the form:

$$
d f(t, T)=\mu_{f}(t, T) d t+\sigma_{f}(t, T) d z_{f}(t)+c_{f}(t, T) d N_{f}(t) \text { given } f(0, T), \quad \forall T \leq T^{*},
$$

with the date- 0 riskless forward curve initialized to the observable values. $T^{*}$ is a distant time horizon, and $z_{f}(t)=\left(z_{1}(t), z_{2}(t), \ldots, z_{m}(t)\right)^{\prime}$ is an $m$-dimensional standard Brownian motion. $N_{f}(t)$ is an independent Poisson process that models jump events, with

$$
d N_{f}(t)= \begin{cases}1 & \text { with probability } \eta_{f} d t \\ 0 & \text { with probability } 1-\eta_{f} d t .\end{cases}
$$

We assume that $\mu_{f}(t, T), \sigma_{f}(t, T)$ and $c_{f}(t, T)$ satisfy standard technical conditions to ensure a strong solution to (2) and well-behaved spot rate and bond price processes. ${ }^{7}$ The volatility structure, $\sigma_{f}(t, T)$, is a predictable $1 \times m$ vector process that at date $t$ depends on observable state variables, while $c_{f}(t, T)$ is a simple deterministic function of time to maturity, $T-t$. The instantaneous spot rate at date $t$ is $r(t)=f(t, t)$.

The dynamics of the bond price follows by applying Ito's lemma for jump-diffusion processes:

$$
\begin{aligned}
\frac{d P(t, T)}{P(t, T)}= & \left(r(t)+\frac{1}{2} \sigma_{p}(t, T) \sigma_{p}^{\prime}(t, T)-\int_{t}^{T} \mu_{f}(t, u) d u\right) d t-\sigma_{p}(t, T) d z_{f}(t) \\
& +\left(e^{-K_{p}(t, T)}-1\right) d N_{f}(t),
\end{aligned}
$$

\footnotetext{
${ }^{7}$ See Heath, Jarrow, and Morton (1992) for the case with no jumps and Bjork, Kabanov, and Runggaldier (1997) for the case with jumps.
} 
where

$$
\begin{aligned}
\sigma_{p}(t, T) & =\int_{t}^{T} \sigma_{f}(t, u) d u \\
K_{p}(t, T) & =\int_{t}^{T} c_{f}(t, u) d u .
\end{aligned}
$$

Now consider a risky zero-coupon corporate bond. Its yield can be broken down into a riskless yield and a credit spread. We first consider firms whose credit spreads do not depend on the default state of other firms. For such a firm, firm A say, that has not defaulted prior to date $t$, we have:

$$
d Y_{A}(t)= \begin{cases}1 & \text { with probability } \eta_{A}(X(t)) d t \\ 0 & \text { with probability } 1-\eta_{A}(X(t)) d t\end{cases}
$$

Firm A's default intensity, $\eta_{A}(X(t))$, could depend on riskless factors as well as on other factors. The time to default, $\tau_{A}$, is a stopping time, and we have $Y_{A}(t)=1_{\left\{\tau_{A} \leq t\right\}}$. With the loss given default being $\ell_{A}(X(t))$, the instantaneous credit spread is given by $\lambda_{A}(t)=\eta_{A}(X(t)) \ell_{A}(X(t))$ and could depend on all state variables in $X(t)$.

Let $\Pi_{A}(t, T)$ represent the date- $t$ price of a bond issued by firm A that promises to pay $\$ 1$ at date $T$. Then,

$$
\Pi_{A}(t, T)=V_{A}(t, T) 1_{\left\{\tau_{A}>t\right\}},
$$

where

$$
\begin{aligned}
V_{A}(t, T) & =e^{-\int_{t}^{T}\left(f(t, u)+\lambda_{A}(t, u)\right) d u} \\
& =P(t, T) S_{A}(t, T) .
\end{aligned}
$$

Here, $f(t, u)$ is the date- $t$ riskless forward interest rate for date $u$, as before, and $\lambda_{A}(t, u)$ is the date- $t$ forward credit spread for firm $\mathrm{A}$ for the future time increment $[u, u+d t]$. The risk-neutral dynamics of the riskless forward rates are given by (2), and the risk-neutral dynamics of the forward credit spreads are given by

$$
d \lambda_{A}(t, T)=\mu_{A}(t, T) d t+\sigma_{A}(t, T) d z_{A}(t)+c_{f A}(t, T) d N_{f}(t), \quad \forall t \leq \tau_{A},
$$

with the date-0 riskless forward curve and the date- 0 firm A credit spread curve initialized to their observable values. The forward credit spreads are driven by a continuous diffusive term, $d z_{A}(t)$, where $z_{A}(t)=\left(z_{A_{1}}(t), \ldots, z_{A_{n}}(t)\right)^{\prime}$ is an $n$-dimensional standard Brownian motion with $E\left(d z_{f}(t) d z_{A}^{\prime}(t)\right)=\Sigma_{m \times n}^{A} d t$. $E$ denotes expectation with respect to the risk-neutral measure, and

$$
\left(\Sigma^{A}\right)_{i j}=\rho_{i j}^{A}
$$

Further, when there is a jump in the riskless curve, then there is a corresponding jump in the 
credit spread curve. We assume that $\mu_{A}(t, T), \sigma_{A}(t, T)$ and $c_{A}(t, T)$ satisfy standard technical conditions to ensure a strong solution to $(7)$ and well-behaved credit spread and risky bond price processes. Similar to the riskless forward rates, the $1 \times n$ volatility factor, $\sigma_{A}(t, T)$, is predictable, while $c_{f A}(t, T)$ is a deterministic function of time to maturity, $T-t$. The instantaneous credit spread at $t$ is $\lambda_{A}(t)=\lambda_{A}(t, t)$.

If there are several firms, then the correlation between their credit spread innovations will presumably be determined by the nature of the operations and the capital structure of the firms. However, the credit spread of any specific firm at any point in time will not be influenced by defaults of any other firm. From a computational point of view then, the price of a zero-coupon bond of such a firm is not dependent on the path of credit spreads of other firms up to that date, but is a function of the riskless yield curve up to that date, as well as of the dynamics of the firm-specific credit spreads.

Application of Ito's lemma for jump-diffusion processes yields:

$$
\begin{aligned}
\frac{d S_{A}(t, T)}{S_{A}(t, T)}= & \left(\lambda_{A}(t)+\frac{1}{2} \sigma_{S_{A}}(t, T) \sigma_{S_{A}}^{\prime}(t, T)-\int_{t}^{T} \mu_{A}(t, u) d u\right) d t-\sigma_{S_{A}}(t, T) d z_{A}(t) \\
& +\left(e^{-K_{f A}(t, T)}-1\right) d N_{f}(t),
\end{aligned}
$$

where

$$
\begin{aligned}
\sigma_{S_{A}}(t, T) & =\int_{t}^{T} \sigma_{A}(t, u) d u, \\
K_{f A}(t, T) & =\int_{t}^{T} c_{f A}(t, u) d u .
\end{aligned}
$$

\section{Proposition 1}

Assume the dynamics of forward rates and forward credit spreads under the risk-neutral measure are given by equations (2) and (7). Suppose that at the time of default of the risky bond, the recovery value is proportional to the market value of the bond just prior to default. Then, to avoid arbitrage opportunities, the following drift restrictions must hold:

$$
\begin{aligned}
& \mu_{f}(t, T)=\sigma_{p}(t, T) \sigma_{f}^{\prime}(t, T)-c_{f}(t, T) e^{-K_{p}(t, T)} \eta_{f} \\
& \mu_{A}(t, T)=\sigma_{S_{A}}(t, T) \sigma_{A}^{\prime}(t, T)+\sigma_{f}(t, T) \Sigma^{A} \sigma_{S_{A}}^{\prime}(t, T)+\sigma_{p}(t, T) \Sigma^{A} \sigma_{A}^{\prime}(t, T)+g_{A}(t, T)
\end{aligned}
$$

where

$$
g_{A}(t, T)=\eta_{f}\left(c_{f}(t, T) e^{-K_{p}(t, T)}-\left(c_{f}(t, T)+c_{f A}(t, T)\right) e^{-\left(K_{p}(t, T)+K_{f A}(t, T)\right)}\right) .
$$

Proof: See Appendix A.

Equations (10) and (11) curtail the drift expressions in terms of the volatility structures. For 
the case with no jumps, the restriction on the drift terms for riskless forward rates under the riskneutral measure was first identified by Heath, Jarrow, and Morton (1992), and the corresponding restrictions for risky forward rates were identified by several authors, including Schönbucher (2000). In comparison, the above equations incorporate jumps in riskless yields and credit spread curves. The dynamics of riskless and risky bond prices are not Markovian in a finite collection of state variables. This creates computational difficulties since the entire riskless and risky term structures have to be stored along all the paths that are generated.

\section{Markovian Models For Defaultable Bonds}

We now impose modest restrictions on volatilities and jumps that enable us to obtain low-dimensional Markovian representations for the term structures of riskless interest rates and credit spreads. The constraints will leave free the specification of the volatilities of instantaneous riskless rate and instantaneous credit spread for each name. Instead, the constraints will involve the volatilities of forward rates relative to their spot rates.

Specifically, we need to curtail the volatility structures $\sigma_{f}(t, T)=\left(\sigma_{f_{1}}(t, T), \ldots, \sigma_{f_{m}}(t, T)\right)$ and the impact factor $c_{f}(t, T)$ for riskless debt, as well as $\sigma_{A}(t, T)=\left(\sigma_{A_{1}}(t, T), \ldots, \sigma_{A_{n}}(t, T)\right)$ and $c_{f A}(t, T)$ for risky debt as follows:

$$
\begin{aligned}
\sigma_{f_{i}}(t, T) & =h_{f_{i}}(t) e^{-\kappa_{f_{i}}(T-t)}, \quad i=1, \ldots, m, \\
\sigma_{A_{j}}(t, T) & =h_{A_{j}}(t) e^{-\kappa_{A_{j}}(T-t)}, \quad j=1, \ldots, n .
\end{aligned}
$$

Here, $h_{f_{i}}(t)$ and $h_{A_{j}}(t)$ are the loadings that determine the volatility of the instantaneous riskless rate and credit spread, respectively. They are predictable functions that depend on state variables at date $t$, and are subject only to standard technical conditions that ensure strong solutions to (2) and (7). We require the jump impact factors to be of the form:

$$
\begin{aligned}
c_{f}(t, T) & =c_{f} e^{-\gamma_{f}(T-t)}, \\
c_{f A}(t, T) & =c_{f A} e^{-\gamma_{f A}(T-t)} .
\end{aligned}
$$

Substituting these expressions into equations (4), (5), (8) and (9), the volatility expressions $\sigma_{p}(t, T)=\left(\sigma_{p_{1}}(t, T), \ldots, \sigma_{p_{m}}(t, T)\right)$ and $\sigma_{A}(t, T)=\left(\sigma_{A_{1}}(t, T), \ldots, \sigma_{A_{n}}(t, T)\right)$ are given by:

$$
\begin{aligned}
\sigma_{p_{i}}(t, T) & =h_{f_{i}}(t) K\left(t, T ; \kappa_{f_{i}}\right), \quad i=1, \ldots, m, \\
\sigma_{S_{A_{j}}}(t, T) & =h_{A_{j}}(t) K\left(t, T ; \kappa_{A_{j}}\right), \quad j=1, \ldots, n,
\end{aligned}
$$


where $K(t, T ; x)=\frac{1}{x}\left(1-e^{-x(T-t)}\right)$. The impact factors $K_{p}(t, T)$ and $K_{f A}(t, T)$ are computed as:

$$
\begin{aligned}
K_{p}(t, T) & =c_{f} K\left(t, T ; \gamma_{f}\right), \\
K_{f A}(t, T) & =c_{f A} K\left(t, T ; \gamma_{f A}\right) .
\end{aligned}
$$

With these volatility and impact restrictions, Markovian models with exponential affine riskless and risky bond prices can be obtained.

\section{Proposition 2}

(i) Under the risk-neutral dynamics (2), with the volatility and impact restrictions given in (13) and (15), the riskless bond price at date $t$ is linked to the forward price of the bond at date 0 by:

$P(t, T)=\frac{P(0, T)}{P(0, t)} \exp \left(-\sum_{i=1}^{m} H_{1, i}(t, T) \psi_{1, i}(t)-\sum_{i=1}^{m} H_{2, i}(t, T) \psi_{2, i}(t)-H_{3}(t, T) \psi_{3}(t)+H_{J}(t, T)\right)$,

where

$$
\begin{aligned}
H_{1, i}(t, T) & =\frac{1}{\kappa_{f_{i}}} K\left(t, T ; \kappa_{f_{i}}\right), \quad i=1, \ldots, m, \\
H_{2, i}(t, T) & =-\frac{1}{\kappa_{f_{i}}} K\left(t, T ; 2 \kappa_{f_{i}}\right), \quad i=1, \ldots, m, \\
H_{3}(t, T) & =c_{f} K\left(t, T ; \gamma_{f}\right), \\
H_{J}(t, T) & =c_{f} \eta_{f} \int_{t}^{T} L_{f}(t, u) d u .
\end{aligned}
$$

and

$$
L_{f}(t, T)=\frac{1}{c_{f}}\left(e^{-c_{f} K\left(t, T ; \gamma_{f}\right)}-e^{-c_{f} K\left(0, T ; \gamma_{f}\right)}\right) .
$$

The dynamics of the state variables, initialized to 0 at date 0 , are given by:

$$
\begin{aligned}
d \psi_{1, i}(t) & =\left(h_{f_{i}}^{2}(t)-\kappa_{f_{i}} \psi_{1, i}(t)\right) d t+\kappa_{f_{i}} h_{f_{i}}(t) d z_{f_{i}}(t), \\
d \psi_{2, i}(t) & =\left(h_{f_{i}}^{2}(t)-2 \kappa_{f_{i}} \psi_{2, i}(t)\right) d t \\
d \psi_{3}(t) & =-\gamma_{f} \psi_{3}(t) d t+d N_{f}(t) .
\end{aligned}
$$

(ii) Given the risk-neutral dynamics (2) and (7), the volatility and impact structures specified in (13) through (16), and assuming that at the time of default, the recovery value is proportional to the market value of the bond just prior to default, the price of a defaultable zero-coupon bond is given 
by $\Pi_{A}(t, T)=V_{A}(t, T) 1_{\left\{\tau_{A}>t\right\}}$, where $V_{A}(t, T)=P(t, T) S_{A}(t, T)$ and

$$
\begin{aligned}
S_{A}(t, T)= & \frac{S_{A}(0, T)}{S_{A}(0, t)} \exp \left(-A_{0}(t, T)-\sum_{j=1}^{n}\left(K_{0, j}(t, T) \xi_{0, j}-K_{1, j}(t, T) \xi_{1, j}\right)\right) \\
& \times \exp \left(\sum_{i=1}^{m} \sum_{j=1}^{n}\left(K_{2, i j}(t, T) \xi_{2, i j}-K_{3, i j}(t, T) \xi_{3, i j}-K_{4, i j}(t, T) \xi_{4, i j}\right)-K_{5}(t, T) \xi_{5}(t)\right) .
\end{aligned}
$$

Here, $A_{0}(t, T)=\int_{t}^{T} G_{A}(t, u) d u$, where $g_{A}$ is defined in (12) and $G_{A}(t, u)=\int_{0}^{t} g_{A}(v, u) d v$, and

$$
\begin{aligned}
K_{0, j}(t, T) & =\frac{1}{\kappa_{A_{j}}} K\left(t, T ; \kappa_{A_{j}}\right), \quad j=1, \ldots, n, \\
K_{1, j}(t, T) & =\frac{1}{\kappa_{A_{j}}} K\left(t, T ; 2 \kappa_{A_{j}}\right), \quad j=1, \ldots, n, \\
K_{2, i j}(t, T) & =\frac{\rho_{i j}^{A}\left(\kappa_{f_{i}}+\kappa_{A_{j}}\right)}{\kappa_{f_{i}} \kappa_{A_{j}}} K\left(t, T ; \kappa_{f_{i}}+\kappa_{A_{j}}\right), \quad i=1, \ldots, m, j=1, \ldots, n, \\
K_{3, i j}(t, T) & =\frac{\rho_{i j}^{A}}{\kappa_{A_{j}}} K\left(t, T ; \kappa_{f_{i}}\right), \quad i=1, \ldots, m, j=1, \ldots, n, \\
K_{4, i j}(t, T) & =\frac{\rho_{i j}^{A}}{\kappa_{f_{i}}} K\left(t, T ; \kappa_{A_{j}}\right), \quad i=1, \ldots, m, j=1, \ldots, n, \\
K_{5}(t, T) & =c_{f A} K\left(t, T ; \gamma_{f A}\right) .
\end{aligned}
$$

The dynamics of the state variables, all initialized at date 0 to be 0 , are given by:

$$
\begin{aligned}
d \xi_{0, j}(t) & =\left(h_{A_{j}}^{2}(t)-\kappa_{A_{j}} \xi_{0, j}(t)\right) d t+\kappa_{A_{j}} h_{A_{j}}(t) d z_{A_{j}}(t) \\
d \xi_{1, j}(t) & =\left(h_{A_{j}}^{2}(t)-2 \kappa_{A_{j}} \xi_{1, j}(t)\right) d t \\
d \xi_{2, i j}(t) & =\left(h_{f_{i}}(t) h_{A_{j}}(t)-\left(\kappa_{A_{j}}+\kappa_{f_{i}}\right) \xi_{2, i j}(t)\right) d t \\
d \xi_{3, i j}(t) & =\left(h_{f_{i}}(t) h_{A_{j}}(t)-\kappa_{f_{i}} \xi_{3, i j}(t)\right) d t \\
d \xi_{4, i j}(t) & =\left(h_{f_{i}}(t) h_{A_{j}}(t)-\kappa_{A_{j}} \xi_{4, i j}(t)\right) d t \\
d \xi_{5}(t) & =-\gamma_{f A} \xi_{5}(t)+d N_{f}(t) .
\end{aligned}
$$

Proof: See Appendix A.

This Proposition is a main theoretical contribution of our paper. It delivers exponential affine riskless and risky bond prices. This is perhaps surprising given that the instantaneous covariance matrix is not necessarily an affine function of the state variables. Indeed, the volatility loadings, $h_{f}(t)$ and $h_{A}(t)$, can be chosen from a wide range of functions, and are subject only to standard technical conditions that ensure strong solutions exist for the stochastic differential equations. This feature greatly expands the possible choice of volatility structures that can be used to price derivatives. It comes at a small cost of having to carry a few path statistics that allow a low-level 
Markovian representation.

As an example, the volatility of spot interest rates and credit spreads could be specified as

$$
\begin{aligned}
h_{f}(t) & =\min \left(\left|\tilde{h}_{f}(t)\right|, \bar{h}_{f}\right), \\
h_{A}(t) & =\min \left(\left|\tilde{h}_{A}(t)\right|, \bar{h}_{A}\right),
\end{aligned}
$$

for large yet finite constants $\bar{h}_{f}$ and $\bar{h}_{A}$. In particular, $\tilde{h}_{f}(t)$ and $\tilde{h}_{A}(t)$ could be affine in the state variables. Since the spot and forward rates are affine functions of the state variables given by (A.2) and (A.3) in the appendix, this means that the volatilities could be affine functions of points on the forward riskless yield or credit spread curves, a specification that clearly falls outside the DuffieKan class of models. While not always necessary, to prevent explosive processes and to ensure the resulting stochastic differential equations have strong solutions, we place bounds on the volatility structures.

The first part of the Proposition shows that, given an initial riskless term structure, riskless bond prices at any future date are fully characterized by $2 m+1$ state variables, namely $\left(\psi_{1}(t), \psi_{2}(t), \psi_{3}(t)\right)$, where $\psi_{1}(t)$ and $\psi_{2}(t)$ are of size $m$. When interest rates are driven by one stochastic driver $(m=1)$ with no jumps, the model reduces to Ritchken and Sankarasubramanian (1995). For the slightly more general case of $m>1, n=0$ and no jumps, the model corresponds to that of Inui and Kijima (1998). With interest rate jumps we get a modest extension. And when $n$ is released from zero, we get a large new set of tractable models for risky bond prices.

The second part of Proposition 2 states that forward credit spreads of all maturities at date $t$ are linked to the credit spread curve at date 0 through a total of at most $2 n+3 m n+1$ state variables. Risky bond prices are determined by these $2 n+3 m n+1$ state variables in addition to the $2 m+1$ state variables for the riskless factors. Collectively then, the vector $X(t)$ consists of at most $3 m n+2(m+n+1)$ state variables. Note, however, that these $3 m n+2(m+n+1)$ state variables are driven by a much smaller set of $m+n$ Brownian motions. This is crucial, as it limits the computational burden of estimating parameters for our proposed class of models to that of $m+n$-dimensional affine models with jumps. For example, if $m=3$ and $n=2$, the total number of state variables is at most 30 . The estimation issues of such a model are comparable to an affine model with five sources of uncertainty.

To illustrate the benefits of Proposition 2, consider a problem where cash flows occur monthly over a 10-year time horizon, and that the terminal cash flow of some credit derivative depends on the riskless and risky discount function going out another 20 years. In a typical HJM model, the forward rates of $30 \times 12=360$ monthly interest rates as well as 360 credit spreads would need to be tracked. As such, the model is Markovian in 720 state variables. If the time partitions are refined to weeks, then weekly forward rates and spreads must be computed and the number of state variables increases by a factor of four to 2,880. In contrast, with our Markovian models, a maximum of nine state variables need to be maintained, no matter how much the partition is refined.

To further highlight the computational effort involved with our models, consider the simple 
specification with two stochastic drivers, one for interest rates $(m=1)$ and one for credit spreads $(n=1)$, and no jumps.

\section{Corollary to Proposition 2}

For the case $m=n=1$ and no jumps, equivalent representations for riskless bond prices, $P(t, T)$, and risky bond prices, $\Pi_{A}(t, T)=V_{A}(t, T) 1_{\left\{\tau_{A}>t\right\}}$, where $V_{A}(t, T)=P(t, T) S_{A}(t, T)$, are:

$$
\begin{aligned}
P(t, T) & =\frac{P(0, T)}{P(0, t)} \exp \left(-K\left(t, T ; \kappa_{f}\right)(r(t)-f(0, t))-\frac{1}{2} K^{2}\left(t, T ; \kappa_{f}\right) \psi(t)\right) \\
S_{A}(t, T) & =\frac{S_{A}(0, T)}{S_{A}(0, t)} \exp \left(-K\left(t, T ; \kappa_{A}\right)\left(\lambda_{A}(t)-\lambda_{A}(0, t)\right)-\sum_{j=1}^{3} K_{j}(t, T) \xi_{j}(t)\right) .
\end{aligned}
$$

The terms in the exponent of $S_{A}(t, T)$ are given as:

$$
\begin{aligned}
K_{1}(t, T) & =\frac{1}{2} K\left(t, T ; \kappa_{A}\right)^{2} \\
K_{2}(t, T) & =\rho^{A}\left(\frac{1}{\kappa_{f}}+\frac{1}{\kappa_{A}}\right)\left(K\left(t, T ; \kappa_{A}\right)-K\left(t, T ; \kappa_{f}+\kappa_{A}\right)\right), \\
K_{3}(t, T) & =\frac{\rho^{A}}{\kappa_{A}}\left(K\left(t, T ; \kappa_{f}\right)-K\left(t, T ; \kappa_{A}\right)\right) .
\end{aligned}
$$

The dynamics of the state variables are given by:

$$
\begin{aligned}
d r(t) & =\mu_{r}(t) d t+h_{f}(t) d z_{f}(t), \\
d \lambda_{A}(t) & =\mu_{\lambda_{A}}(t) d t+h_{A}(t) d z_{A}(t),
\end{aligned}
$$

together with the path statistics:

$$
\begin{aligned}
d \psi(t) & =\left(h_{f}^{2}(t)-2 \kappa_{f} \psi(t)\right) d t \\
d \xi_{1}(t) & =\left(h_{A}^{2}(t)-2 \kappa_{A} \xi_{1}(t)\right) d t \\
d \xi_{2}(t) & =\left(h_{f}(t) h_{A}(t)-\left(\kappa_{f}+\kappa_{A}\right) \xi_{2}(t)\right) d t \\
d \xi_{3}(t) & =\left(h_{f}(t) h_{A}(t)-\kappa_{f} \xi_{3}(t)\right) d t .
\end{aligned}
$$

The drift terms are given by

$$
\begin{aligned}
\mu_{r}(t) & =\kappa_{f}(f(0, t)-r(t))+\frac{d}{d t} f(0, t)+\psi(t) \\
\mu_{\lambda_{A}}(t) & =\kappa_{A}\left(\lambda_{A}(0, t)-\lambda_{A}(t)\right)+\frac{d}{d t} \lambda_{A}(0, t)+\sum_{j=1}^{3} k_{j} \xi_{j}+\rho^{A} h_{f}(t) h_{A}(t),
\end{aligned}
$$

where $k_{1}=1, k_{2}=\rho^{A}\left(\frac{\kappa_{f}}{\kappa_{A}}+1\right), k_{3}=\rho^{A}\left(1-\frac{\kappa_{f}}{\kappa_{A}}\right)$. 
Proof: See Appendix A.

For the above model, the number of state variables drops to six. Four of these $\left(\psi(t), \xi_{1}(t), \xi_{2}(t)\right.$ and $\left.\xi_{3}(t)\right)$ are simply path statistics, that is, deterministic functions conditional on sample paths for $r$ and $\lambda_{A}$. When interest rates and credit spreads are uncorrelated, the number of state variables drops to four as $\xi_{2}(t)$ and $\xi_{3}(t)$ are no longer needed. Finally, if the predictable functions, $h_{f}(t)$ and $h_{A}(t)$, are constants, then all path statistics fall away, and the generalized Vasicek (1977) models for both riskless and risky bond prices obtains, with just the two state variables $r(t)$ and $\lambda_{A}(t)$.

The state variables for the riskless term structure in these simple model are $\Phi(t)$, say, where $\Phi(t)=\{r(t), \psi(t)\}$. The state variables for the price of a bond issued by firm A is $X_{A}(t)$, say, where $X_{A}(t)=\left\{\Phi(t), \Upsilon_{A}(t)\right\}$. Here, $\Upsilon_{A}(t)=\left\{\lambda_{A}(t), \xi_{1}(t), \xi_{2}(t), \xi_{3}(t)\right\}$ are the additional state variables for the credit spreads.

An attractive feature of the Markovian HJM models is that we can easily map the path statistics to unique points on the term structure. As an illustration, we again consider the simple specification with two stochastic drivers $(m=n=1)$ and no jumps. From equation (19), we obtain the forward rate expression for date $T=t+s$ as:

$$
f(t, t+s)=f(0, t+s)+e^{-\kappa_{f} s}(r(t)-f(0, t))+\frac{e^{-\kappa_{f} s}}{\kappa_{f}}\left(1-e^{-\kappa_{f} s}\right) \psi(t) .
$$

(For details, see equation (A.4) with $T=t+s$ in Appendix A.) We can then replace the path statistic $\psi(t)$ with the forward rate $f(t, t+s)$. Other forward rates can be expressed as affine combinations of the new state variables, $r(t)$ and $f(t, t+s)$. In particular, for maturity $t+\tilde{s}$,

$$
\begin{aligned}
f(t, t+\tilde{s})= & f(0, t+\tilde{s})+\left(e^{-\kappa_{f} \tilde{s}}-W\left(s, \tilde{s} ; \kappa_{f}\right)\right)(r(t)-f(0, t)) \\
& +W\left(s, \tilde{s} ; \kappa_{f}\right)(f(t, t+s)-f(0, t+s))
\end{aligned}
$$

where $W\left(s, \tilde{s} ; \kappa_{f}\right)=\frac{1-e^{-\kappa_{f} \tilde{s}}}{1-e^{-\kappa_{f}}}$. Hence changes in all forward rates can be expressed as functions of changes in two forward rate points on the curve. ${ }^{8}$ This is in stark contrast with the general (non-Markovian) HJM models that require all points on the forward rate curve be carried.

Similarly, for the credit spread curve, equation (20) for $T=t+s_{i}$ yields

$$
\lambda_{A}\left(t, t+s_{i}\right)=\lambda_{A}\left(0, t+s_{i}\right)+e^{-\kappa_{A} s_{i}}\left(\lambda_{A}(t)-\lambda_{A}(0, t)\right)+\sum_{j=1}^{3} D_{j}\left(s_{i}, \kappa_{A}, \kappa_{f}, \rho^{A}\right) \xi_{j}(t),
$$

where $D_{j}\left(s_{i}, \kappa_{A}, \kappa_{f}, \rho^{A}\right)$ are the coefficients of $\xi_{j}(t)$ which are provided in the appendix as the coefficients in equation (A.5). The three state variables $\xi_{j}(t), j=1,2,3$, can therefore be mapped onto three forward rates, with maturities $s_{1}, s_{2}, s_{3}$. Hence, all points on the credit spread curve can be represented as maturity-dependent combinations of just four points on the credit curve. This,

\footnotetext{
${ }^{8}$ For more details of transforming the state variables to points on the forward curve, see Bliss and Ritchken (1996), and especially Chiarella and Kwon (2002).
} 
again, is in contrast to the more general HJM model that requires all points on the credit curve.

Are the Volatility Restrictions Severe? The restrictions that permit a Markovian representation assume that forward rate volatilities of increasing maturities, normalized by the spot rate volatility, are exponentially decaying with maturity. Empirical evidence suggests that there could be a hump in the volatility structure of forward rates. ${ }^{9}$ This feature can easily be accommodated in our multifactor models $(m>1, n>1)$. In addition, Proposition 2 can be generalized to enable humped volatility structures even when $m=n=1$. In fact, we are able to establish arbitrary shapes in the term structure of volatilities. For example, in a single-factor model for riskless forward rates, we could replace (13) by:

$$
\sigma_{f}(t, T)=h_{f}(t) \sum_{i=1}^{k} a_{i} e^{-\kappa_{i}(T-t)}, \quad k>1,
$$

which permits humped structures for volatilities. To illustrate this, consider the case where $k=2$. Substituting equation (27) into the HJM drift restriction (10), using the resulting expression in equation (2), and then integrating leads to:

$$
\begin{aligned}
f(t, T)= & f(0, T)+\sum_{i=1}^{2} e^{-\kappa_{i}(T-t)} \psi^{(i)}(t)-\sum_{i=1}^{2} \frac{a_{i}^{2}}{\kappa_{i}} e^{-2 \kappa_{i}(T-t)} \psi^{(i, i)}(t) \\
& -a_{1} a_{2}\left(\frac{1}{\kappa_{1}}+\frac{1}{\kappa_{2}}\right) e^{-\left(\kappa_{1}+\kappa_{2}\right)(T-t)} \psi^{(1,2)}(t)
\end{aligned}
$$

where the dynamics of the state variables are given by:

$$
\begin{aligned}
d \psi^{(i)}(t) & =\left(a_{i}\left(\frac{a_{1}}{\kappa_{1}}+\frac{a_{2}}{\kappa_{2}}\right) h_{f}^{2}(t)-\kappa_{i} \psi^{(i)}\right) d t+a_{i} h_{f}(t) d z_{f}(t), \quad i=1,2, \\
d \psi^{(i, j)}(t) & =\left(h_{f}^{2}(t)-\left(\kappa_{i}+\kappa_{j}\right) \psi^{(i, j)}(t)\right) d t, \quad i, j=1,2 .
\end{aligned}
$$

Compared to our single-factor model of forward riskless rates in the Corollary to Proposition 2, the entire term structure requires three additional state variables, while keeping the number of stochastic drivers at $m=1$. The volatility parameters $a_{i}$ and $\kappa_{i}, i=1,2$, can easily be chosen so as to closely match the potentially humped volatility term structure of forward rates. In a similar way, the volatility term structures of credit spreads can be generalized to permit humps as well.

Relationship with Duffie-Kan Affine Models. To set our results in perspective, we explain the relationship between our framework and that of Duffie and Kan (1996). Focusing on riskless bonds, the latter specify the spot rate as an affine function of some $m$-dimensional state vector, $X(t)$, as $r(t)=\delta_{0}+\delta_{1}^{\prime} X(t)$. The risk-neutral dynamics of the state vector are given by $d X(t)=$

\footnotetext{
${ }^{9}$ For discussions of this issue, see Litterman and Scheinkman (1991), Heath, Jarrow, Morton, and Spindel (1992), Amin and Morton (1994), Goncalves and Issler (1996) and Brigo and Mercurio (2001).
} 
$\mu_{X}(t) d t+\sigma_{X}(t) d z(t)$, where $\mu_{X}(t)=\left(\mu_{X_{1}}(t), \ldots, \mu_{X_{m}}(t)\right)^{\prime}$ and $\sigma_{X}(t)=\left(\sigma_{X_{1}}(t), \ldots, \sigma_{X_{m}}(t)\right)^{\prime}$. Bond prices are computed as $P(t, T)=E_{t} e^{-\int_{t}^{T} r(u) d u}$. Duffie and Kan find that a sufficient condition for the bond price to be exponential affine in the state variables is that the riskneutral drift and instantaneous variance of the state vector are affine in $X(t)$. To derive necessary conditions, they observe that if $P(t, T)=e^{A(t, T)+B(t, T) X(t)}$ for some functions $A(t, T)$ and $B(t, T)=\left(B_{1}(t, T), \ldots, B_{m}(t, T)\right)^{\prime}$ with $A(t, t)=0$ and $B(t, t)=0$, the following fundamental PDE has to be satisfied:

$$
a(X(t), t, T)=\sum_{i=1}^{m} B_{i}(t, T) \mu_{X_{i}}(t)+\frac{1}{2} \sum_{i} \sum_{j} B_{i}(t, T) B_{j}(t, T) \sigma_{X_{i}}(t) \sigma_{X_{j}}^{\prime}(t),
$$

where $a(X(t), t, T)=r(t)-A_{t}(t, T)-B_{t}^{\prime}(t, T) X(t)$ and subscripts on $A$ and $B$ represent derivatives. This is equation (3.6) in Duffie and Kan (1996), which implies that a necessary condition for exponential affine bond prices is the existence of $N=m+\frac{m(m+1)}{2}$ maturities $t_{1}, \ldots, t_{N}$, with $t<t_{1}<\ldots<t_{N}$ and a $N \times N$ matrix $C\left(t_{1}, \ldots, t_{N}\right)$ such that

$$
C\left(t_{1}, \ldots, t_{N}\right)\left(\begin{array}{c}
\mu_{X}^{\prime}(t) \\
\sigma_{X_{1}}(t) \sigma_{X_{1}}^{\prime}(t) \\
\sigma_{X_{1}}(t) \sigma_{X_{2}}(t) \\
\ldots \\
\sigma_{X_{m}}(t) \sigma_{X_{m}}^{\prime}(t)
\end{array}\right)=\left(\begin{array}{c}
r(t)-A_{t}\left(t, t_{1}\right)-B_{t}^{\prime}\left(t, t_{1}\right) X(t) \\
\ldots \\
r(t)-A_{t}\left(t, t_{N}\right)-B_{t}^{\prime}\left(t, t_{N}\right) X(t)
\end{array}\right)
$$

where only $\sigma_{X_{i}}(t) \sigma_{X_{j}}^{\prime}(t)$ with $i \leq j$ are included on the left-hand side. The necessary condition for exponential affine bond prices in Duffie and Kan assumes that $C=C\left(t_{1}, \ldots, t_{N}\right)$ can be chosen to be non-singular. Clearly, if that is the case, we can multiply both sides in (29) by $C^{-1}$ to obtain $\mu_{X}$ and $\sigma_{X} \sigma_{X}^{\prime}$ as affine functions of $X(t)$. But if such a non-singular $C$ matrix does not exist, then the necessary condition does not need to hold.

For our family of models, $C$ is indeed singular. To see this, consider the simplest case where there is one stochastic driver and no jumps. In the notation of the Corollary to Proposition 2, the state vector $X(t)=(r(t), \psi(t))^{\prime}$ has risk-neutral dynamics:

$$
\begin{aligned}
d r(t) & =\mu_{r}(t) d t+h_{f}(t) d z_{f}(t), \\
d \psi(t) & =\mu_{\psi}(t) d t .
\end{aligned}
$$

The fundamental PDE in (28) can be written as

$$
a(X(t), t, T)=B_{1}(t, T) \mu_{r}(t)+B_{2}(t, T) \mu_{\psi}(t)+\frac{1}{2} B_{1}^{2}(t, T) h_{f}^{2}(t),
$$

where $a(X(t), t, T)=r(t)-A_{t}(t, T)-B_{1 t}^{\prime}(t, T) r(t)-B_{2 t}^{\prime}(t, T) \psi(t)$. Since $a(X(t), t, T)$ is affine in $X(t)$, the same has to be true for the right-hand side in equation (30). And as long as (i) $B_{2}(t, T)=-\frac{1}{2} B_{1}^{2}(t, T)$ and (ii) $\mu_{r}(t)$ and $\mu_{\psi}(t)-h_{f}^{2}(t)$ are affine in $X(t)$, that is indeed the 
case, whether or not the instantaneous variance $h_{f}^{2}(t)$ is affine in $X(t)$. Both (i) and (ii), as well as (30), are clearly satisfied in the Corollary to Proposition 2, where, with $f(0, t)=\theta_{f}, \mu_{r}(t)=$ $\kappa_{f}\left(\theta_{f}-r(t)\right)+\psi(t), \mu_{\psi}(t)=h_{f}^{2}(t)-2 \kappa \psi(t), B_{1}(t, T)=-K\left(t, T ; \kappa_{f}\right), B_{2}(t, T)=-\frac{1}{2} K\left(t, T ; \kappa_{f}\right)^{2}$ and $A(t, T)=\theta\left(K\left(t, T ; \kappa_{f}\right)-(T-t)\right)$. But as long as $B_{2}(t, T)=-\frac{1}{2} B_{1}^{2}(t, T)$ holds, the second and third column of $C$ in equation (29) are perfectly correlated, and hence $C$ is singular for any choice of maturities.

The above analysis highlights the fact that our family of models is not inconsistent with Duffie and Kan, but rather complements their results. Our models are built on different underlying stochastic processes, where the number of state variables is larger than the number of stochastic drivers, and the drift terms of the path statistics offset spot rate volatilities in a manner that allows bond yields to be affine in the states, even though the state variables themselves do not have to be affine processes. As a result, the family of models we have established are very rich in structure, yet are easy to implement.

\section{Empirical Evidence and Sensitivity Analysis}

This section describes a road map for empirical work for our proposed class of models, and provides an estimation example for a specific model satisfying the Corollary of Proposition 2. We also perform a sensitivity analysis that highlights the importance of incorporating the interaction between credit spreads and interest rates into our model structure using two additional examples.

\subsection{Empirical Evidence}

The majority of empirical studies on the term structure of riskless rates have been conducted within the affine, and to a lesser degree the quadratic, families. ${ }^{10}$ Much fewer studies have focused on the Markovian HJM family. Examples include the cross sectional studies by Fan, Gupta, and Ritchken (2003 and 2007) on Markovian HJM models for riskless rates. While the former study uses cap and swaption data and shows that Markovian models can explain the volatility skew in derivatives markets very well, the latter argues that a one-factor multi-state-variable Markovian HJM model can price caps and swaptions as well as four-factor models where the volatility structure is identified from a principal component analysis as in Longstaff, Santa-Clara, and Schwartz (2001).

Studies that exploit information not only in the cross section but also the time series of bond yields necessarily rely on specifications under both the physical and risk-neutral measures, and are more likely to empirically discriminate between models. There are a few studies of Markovian HJM models for riskless rates that are carried out along these dimensions. For example, De Jong and Santa-Clara (1999) find that their one-factor two-state HJM Markovian models outperform one-factor affine term structure models as proposed by Duffie and Kan (1996). Chiarella, Hung,

\footnotetext{
${ }^{10}$ See Dai and Singleton (2003) for a detailed survey on dynamic term structure models, and Piazzesi (2003) for a survey paper on the use of affine term structure models in macroeconomics.
} 
and To (2009) develop an estimation framework that can be applied to the class of Markovian HJM models. They examine several multifactor models in international markets, with extremely promising results.

Empirical studies on the term structure of credit spreads have come under less scrutiny than the term structure of riskless rates. Theoretical option models, starting with Merton (1974), Longstaff and Schwartz (1995) and Jarrow, Lando, and Turnbull (1997), among others, permit credit spread curves to be increasing, decreasing or hump-shaped. ${ }^{11}$ Given the increased liquidity over the past five years of the credit default swap (CDS) market, where insurance against default is traded, firmspecific credit spread curves can now be easily identified. Further, similar to riskless rates, the term structure of credit spread volatilities can be analyzed.

In this section, we explain how the parameters of our family of models can be readily estimated using full information on both the time series and cross section of riskless yields and credit spreads. The top panel of Figure 1 shows the time series of weekly (Wednesday) riskless interest rates, for maturities ranging from one to ten years. The data is provided by Gurkaynak, Sack, and Wright (2006). Our sample covers the period from February 1, 2005 until May 15, 2009. The median 5 -year riskless rate during that period was $4.1 \%$. The bottom panel shows the time series of credit spreads for two large U.S. firms, in this case, AMR and Lennar. Credit spreads are measured as at-market default swap rates, which are provided by Credit Market Analysis, Inc. (CMA). AMR Corporation is the parent company of American Airlines, the second largest airline in the world, and Lennar is one of the top two homebuilders in the U.S. During the sample period, AMR and Lennar had a median S\&P long-term credit rating of $\mathrm{B}$ and $\mathrm{BBB}$, respectively, representing the high- and medium-credit-quality spectrum of the CDS market. The median 5-year CDS rates were $10.8 \%$ (AMR) and 88 basis points (Lennar). ${ }^{12}$

\section{Figure 1 Here}

The plots clearly reveal significant time variation in the levels and shapes of the riskless yield and credit spread curves. Interest rates and credit spreads generally move in opposite directions, while we observe positive correlations between changes in credit spreads of the two firms. Interestingly, the correlations among changes in riskless yields of different maturities are lower than the correlations among credit spread changes of the same maturities for the two firms, indicating that the former are driven by a larger number of stochastic factors.

\footnotetext{
${ }^{11}$ Empirical studies conducted to investigate the shape of the term structure of corporate bond yield spreads include Sarig and Warga (1989), Fons (1994), Bohn (1999), Helwege and Turner (1999), He, Hu, and Lang (2004) and Bedendo, Cathcart, and El-Jahel (2007).

${ }^{12}$ Unlike most of the other legacy airlines, AMR has not filed for bankruptcy, has consequently not been able to restructure itself under protection from creditors, and, as a result of especially high labor and pension costs, has been at a competitive disadvantage. AMR's debt is rated speculative grade, and its credit spreads have been extremely high, even prior to the current financial crisis. Lennar, on the other hand, had investment-grade debt prior to the subprime crisis, but has been downgraded to BB since. Both firms have liquid CDS contracts and are constituents of the Markit CDX index family: AMR is in the high-yield index (CDX.NA.HY) while Lennar used to be part of the investment-grade index (CDX.NA.IG), but is now a member of the crossover index (CDX.NA.XO).
} 
We use the data to illustrate the estimation procedure for a simple model in the family provided by the Corollary to Proposition 2, but the same procedure carries forward to more general models. For this case, the state variables are:

$$
X(t)=\left(r(t), \lambda_{A}(t), \psi(t), \xi_{1}(t), \xi_{2}(t) \xi_{3}(t)\right)^{\prime}
$$

where $r(t)$ and $\lambda_{A}(t)$ are the two factors that capture instantaneous riskless rates and credit spreads, and $\psi(t), \xi_{1}(t), \xi_{2}(t)$ and $\xi_{3}(t)$ are simple path statistics.

We begin with the risk-neutral dynamics of our six state variables given in equations (21) through (26). Since we use time series data, we also need to specify structures for the market prices of risk. Let $\nu_{f}(t)$ denote the market price of interest rate risk and let $\nu_{A}(t)$ be the price of credit risk orthogonal to interest rate risk. With these assumptions, the dynamics under the data-generating measure are given by:

$$
\begin{aligned}
d r(t) & =\left(\mu_{r}(t)+\nu_{f}(t) h_{f}(t)\right) d t+h_{f}(t) d w_{f}(t) \\
d \lambda_{A}(t) & =\left(\mu_{\lambda_{A}}(t)+\nu_{f}(t) \rho^{A} h_{A}(t)+\nu_{A}(t) \sqrt{1-\rho^{A^{2}}} h_{A}(t)\right) d t+h_{A}(t) d w_{A}(t)
\end{aligned}
$$

where $w_{f}(t)$ and $w_{A}(t)$ are standard Brownian motions with respect to the data-generating measure. The path statistics follow dynamics as in equations (23) to (26). We assume that the market prices of risk are proportional to their spot rate volatilities as:

$$
\begin{aligned}
\nu_{f}(t) & =\nu_{f} h_{f}(t), \\
\nu_{A}(t) & =\nu_{A} h_{A}(t) .
\end{aligned}
$$

For our numerical examples, we choose a model for volatilities that does not overlap with the affine class. In particular, we assume that $\sigma_{f}(t, T)=h_{f}(t) e^{-\kappa_{f}(T-t)}$ and $\sigma_{A}(t, T)=h_{A}(t) e^{-\kappa_{A}(T-t)}$, where $h_{f}(t)$ and $h_{A}(t)$ are as in (17) and (18) with $\tilde{h}_{f}(t)=\sigma_{f} r(t)$ and $\tilde{h}_{A}(t)=\sigma_{A} \lambda_{A}(t)$. The bounds on the spot rate volatilities ensure that the change of measure is arbitrage free. ${ }^{13}$ In a stationary model, the yields on long bonds converge to a constant. Therefore, if considering the steady-state behavior, we can assume the initial forward rate curve to be flat, with $f(0, t)=\theta_{f}$ and, similarly, $\lambda_{A}(0, t)=\theta_{A}$. While not necessary, this simplifies the drift terms in the dynamics of the two state variables $r(t)$ and $\lambda_{A}(t)$.

We estimate the parameters of the model using an extended Kalman filter. The details of the procedure are summarized in Appendix B. The measurement equation is given as:

$$
y(t)=M_{0}(\Theta)+M_{1}(\Theta)^{\prime} X(t)+\varepsilon(t),
$$

\footnotetext{
${ }^{13}$ Heath, Jarrow, and Morton (1992) discuss this approach in the context of their proportional forward rate volatility structure. Amin and Morton (1994) find that ignoring the bounds for this structure has an insignificant effect for valuing derivatives under realistic parameters. We confirm their result in the context of our numerical implementation.
} 
where $y(t)$ is a vector that collects riskless yields and credit spreads of different maturities. While model-implied values for $y(t)$ can be computed from equations (19) and (20), we assume that the data are measured with errors. Measurement errors are assumed to be uncorrelated and normally distributed with mean zero and variance-covariance matrix, $R$ say, with the variance of riskless yield errors being $\sigma_{\epsilon_{f}}^{2}$ and the variance of credit spread errors being $\sigma_{\epsilon_{A}}^{2}$. Given the vector of model parameters,

$$
\Theta=\left\{\theta_{f}, \theta_{A}, \kappa_{f}, \kappa_{A}, \sigma_{f}, \sigma_{A}, \nu_{f}, \nu_{A}, \rho^{A}, \sigma_{\epsilon_{f}}, \sigma_{\epsilon_{A}}\right\},
$$

the form for $M_{0}(\Theta)$ and $M_{1}(\Theta)$ is determined by the Corollary to Proposition 2.

In addition to the measurement equation, we need a discretized transition equation:

$$
X(t+\Delta t)=F_{0}(t, \Theta)+F_{1}(t, \Theta) X(t)+v(t+1)
$$

where $\Delta t$ is the width of the time increment between observations. Under the data-generating measure, conditional on information up to time $t, v(t+1)$ is normally distributed with mean zero and variance-covariance matrix $Q(t, \Theta)$. A detailed derivation of $F_{0}(t, \Theta), F_{1}(t, \Theta)$ and $Q(t, \Theta)$ is provided in Appendix B. These functions depend on the state variables $X(t)$, hence the need for an "extended" Kalman filter.

In our implementation, we first estimate the interest rate parameters $\theta_{f}, \kappa_{f}, \sigma_{f}, \nu_{f}$ and $\sigma_{\epsilon_{f}}$ from the term structure time series data. To estimate the credit spread dynamics using firm-specific data, we follow Duffee (1999) and assume that the riskless parameters estimated in the first phase are the true parameters, and that the values of $r(t)$ and $\psi(t)$ predicted by the Kalman filter recursion are their true values. To estimate the parameters for riskless yields, we include the 1- through 10-year maturity yields into the measurement equation (31). For credit spreads, we rely on the 1-, 3-, 5-, 7- and 10-year CDS rates. To better interpret our findings, we fix $\theta_{f}$ and $\theta_{A}$ at the long-run mean of 1-year riskless yields and credit spreads, respectively.

Table 1 reports the parameter estimates and their Monte Carlo standard errors for riskless rates, credit spreads of AMR and credit spreads of Lennar. All parameter estimates are significantly different from zero, except for the market prices of interest rate and credit risk. These prices are notoriously difficult to estimate (see, for example, De Jong and Santa-Clara (1999)), which calls for alternative structures, perhaps for market prices of risk that are affine in volatilities rather than proportional, to be explored. The top panel of Figure 2 compares the time series of fitted riskless yields with their observed counterparts. The fitted 5 -year yield closely tracks the actual 5-year yield over the entire sample period. However, the fit of the 1-year riskless yield is, on average, biased low with rather poor fits in the middle of the sample period. At the long horizon, the fitted 10-year yield (not shown) closely tracks its actual counterpart until the recent financial crisis. While the one-factor model for riskless rates does a reasonable job, not surprisingly, the fit is not excellent. This performance can be improved by incorporating the volatility hump in equation (27) and/or by adding additional stochastic drivers. 
Table 1 and Figure 2 Here

The middle and bottom panel of Figure 2 show the model-implied credit spreads for AMR and Lennar vis-a-vie their data counterparts. The fits appear to be fairly reasonable across maturities. Note that 5-year credit spreads for AMR fluctuate from a low of 3.35\% in November 2006 to a high of over $56 \%$ in July 2008. Even before the recent financial crisis, AMR was plagued with volatile credit spreads, a fact that is well captured by the model. The extreme volatility during the financial crisis is also well picked up by the model. Disguised, perhaps by the scale, is the fact that the fit is not always excellent. Indeed, there are time periods where the percentage errors in 5 -year credit spreads are as large as 25\%, suggesting that the one-factor model can be improved upon. In contrast to AMR, the credit spreads of Lennar were low and fairly stable prior to Spring 2007. Since then credit spreads have surged. The plots clearly indicate that Lennar's short-term credit spreads were more volatile than the longer-maturity spreads. For the most part, the model spreads closely track the actual values, but like AMR, there were occasions where errors were significant. In summary, the two examples suggest that improvements in the fit can possibly be obtained by adding additional structure to the volatilities of credit spreads, changing the structure for the market price of credit risk, and/or increasing the number of stochastic drivers.

\subsection{The Importance of Interest Rate-Credit Spread Correlations}

We illustrate the importance of incorporating the interaction between credit spreads and interest rates into our model structure with two examples.

\subsubsection{Bond Options}

We simulate the price of a 3-year European call option on a 5-year zero-coupon bond issued by a risky firm, $A$ say. Figure 3 shows the percentage change in the value of the at-the-money option as the correlation between the riskless and the risky diffusive terms, $\rho^{A}$, moves away from zero. ${ }^{14}$ As $\rho^{A}$ becomes negative, as is the usual case in U.S. corporate bond markets (see, for example, Duffee (1999)), the price of the at-the-money option decreases. For our benchmark set of parameters, as $\rho^{A}$ decreases from 0 to -0.9 , option prices decline dramatically by more than twenty percent. The owner of the call option profits from low interest rates and low credit spreads at expiration, which implies that the value of the call option increases as $\rho^{A}$ increases. Note that the sensitivity to $\rho^{A}$ is stronger for out-of-the-money options. It diminishes as the option moves into the money.

Figure 3 Here

\footnotetext{
${ }^{14}$ Based on the empirical evidence in Section 3.1, a benchmark set of parameter values is given by $m=n=1$ and $\kappa_{f}=\kappa_{A}=0.1, \tilde{h}_{f}(t)=r(t), \tilde{h}_{A}(t)=0.5 \lambda_{A}(t)$, and $\bar{h}_{f}=\bar{h}_{A}=10^{6}$. When jumps are permitted, they have a dampening effect on rates as a function of maturity, with $\gamma_{f}=\gamma_{f A}=0.5$. We set the jump size parameter $c_{f}$ equal to -0.01 . We assume there is no recovery at default, and that the initial yield curve is flat at $4 \%$ and the credit spread curve is flat at 5\%. Our Monte Carlo simulations use weekly time steps, and 10,000 sample paths with antithetic sampling.
} 
In Table 2, we compare the sensitivity of bond option prices to $\rho^{A}$ to the sensitivity of the option prices to the jump intensity, $\eta_{f}$, and the impact factor, $c_{f A}$. We find that even when the jump intensity is increased to 0.5 , while keeping $c_{f A}$ at a rather high level of $2.5 \%$, the impact of the shocks are relatively minor. We also document a lack of sensitivity of option prices to an increase in the impact factor, $c_{f A}$. Specifically, we increase $c_{f A}$ to 0.1 , while keeping the jump intensity constant at 100 basis points, with very little change in option prices. The lack of sensitivity of option prices to jump intensities and impact factors is primarily due to the fact that the initial credit spread curves are kept fixed. Therefore, increasing the frequency of jumps, or their impact factors, forces the risk-neutral dynamics to adjust in an offsetting direction so as to keep credit spreads curves in line with their initially observed values.

Table 2 Here

\subsubsection{Insuring Credit Risk in Derivatives Contracts}

The recent crisis has highlighted the importance of appropriately pricing credit risk in all financial transactions. Here we focus on the pricing of counterparty credit risk in a derivatives contract. Specifically, we consider an insurance contract that provides protection on an over-the-counter derivative by ensuring that the instrument will be fully replaced upon the default of the derivative's counterparty. For our numerical implementation, we assume that firm F enters a 5-year floatingfor-fixed interest-rate swap with counterparty B. Firm F is concerned that firm B will default before the maturity of the interest-rate swap contract. It therefore purchases counterparty risk insurance from a third party, firm A, in form of a 5-year contract that stipulates that if firm B defaults within the next five years, the third-party protection seller A pays to firm $\mathrm{F}$ the market value of the floating-for-fixed swap, as long as it is positive at the time of default. In return, $\mathrm{F}$ pays A a quarterly insurance premium until the end of the 5-year term or until default of firm B, whichever occurs first. In this application, we ignore any potential default risk associated with the third-party supplier of the insurance (firm A), but later we reconsider contracts where the insurer may default. Insurance contracts of this form are quite common, and an over-the-counter market for such products, also called contingent credit default swaps (CCDS), has emerged.

To compute the at-market insurance premium, or CCDS rate, assume that the interest-rate swap pays every six months, in arrears. Let $T_{1}=6$ months, $T_{2}=1$ year, etc. until $T_{K}=T$ years. The ex-coupon market value of the swap at some coupon date $T_{i}$ is given by:

$$
\frac{s}{2} \sum_{j=i+1}^{K} P\left(T_{i}, T_{j}\right)-\left(1-P\left(T_{i}, T\right)\right),
$$

where $s$ denotes the annualized at-market 6-month swap rate determined at time 0 . At $T_{i}$, firms $\mathrm{F}$ and $\mathrm{B}$ also pay interest in the amount of $1 / P\left(T_{i-1}, T_{i}\right)-1$ and $s$, respectively. If firm B defaults at time $\tau_{B}, T_{i-1}<\tau_{B}<T_{i}$, then the time- $\tau_{B}$ market value of the interest-rate swap, $W\left(\tau_{B}\right)$, can 
be computed as:

$$
W\left(\tau_{B}\right)=\frac{s}{2} \sum_{j=i}^{K} P\left(\tau_{B}, T_{j}\right)-\left(P\left(\tau_{B}, T_{i}\right)-P\left(\tau_{B}, T\right)\right)-P\left(\tau_{B}, T_{i}\right)\left(\frac{1}{P\left(T_{i-1}, T_{i}\right)}-1\right) .
$$

For sample path $z$, the time-0 value of the protection leg of the CCDS contract is

$$
V_{\text {seller }}^{(z)}=e^{-\int_{0}^{\tau_{B}} r^{(z)}(u) d u} \max \left\{0, W^{(z)}\left(\tau_{B}\right)\right\}
$$

The fair market value of firm F's insurance payments can be computed as $C V_{\text {buyer }}$, where $\mathrm{C}$ is the annualized CCDS rate and

$$
V_{\text {buyer }}=\frac{1}{4} \sum_{j=1}^{4 T} P(0, j / 4) S_{B}(0, j / 4)
$$

The at-market CCDS rate is that choice for the insurance premium $C$ at which the market values of the payments by the buyer and seller of protection are equal.

Table 3 reports simulation results for the CCDS contract. The first two columns show the sensitivity of prices to the correlation between interest rates and credit spreads when jumps are not present. A negative correlation between the riskless term structure and the credit curve implies that at times when default risk is high (and hence the CCDS is likely to trigger payment), interest rates are low, and the value of the swap contract to $\mathrm{F}$ is high. Conversely, if $\rho^{B}$ is positive, then at times when default risk is high, interest rates are high, and the value of the swap to $\mathrm{F}$ is low, possibly negative. As a result, the CCDS rate increases as correlation decreases. The second two columns of the table report the sensitivity of prices to an increase in the jump intensity. We increase $\eta_{f}$ from zero to 0.5 , while keeping the impact factor $c_{f B}$ constant at $2.5 \%$, and find that although greater jump intensities add to the value of the insurance, overall, the impact of increasing intensities is small. Similarly, increasing the impact factor $c_{f B}$ from zero to 0.1 has limited implications for the at-market CCDS rate. Overall, these results echo the results for bond options although, on a relative basis, the impact of $\rho^{B}$ on CCDS rates is much larger.

\section{Table 3 Here}

In summary, our two examples stress the fact that credit-sensitive products can be highly sensitive to the diffusive correlation between interest rates and credit spreads. A unified framework that prices all kinds of credit derivatives therefore needs to be flexible enough to allow for interest rate-credit spread correlations. 


\section{Systemic Credit Shocks and Default Clustering}

We now extend the analysis of the earlier section to more fully incorporate systemic risk features and clustering of defaults. The primary motivation for this extension is to permit meaningful stress tests to be conducted on a portfolio of credits.

So far we incorporate systemic credit shocks by allowing a single market factor to induce an exogenous jump not only in riskless rates, but also in each firm's instantaneous credit spreads. In addition, our models build in correlated defaults by correlating the default intensities. But conditional on the realization of the default intensities, default rates are independent across firms. This conditional independence may prevent us from generating the observed level of default clustering. ${ }^{15}$ The empirical evidence suggests that default contagion of some form is important. Collin-Dufresne, Goldstein, and Helwege (2003) and Jorion and Zhang (2007) argue that a major credit event at one firm may be associated with significant increases in spreads of other firms. ${ }^{16}$

We now extend our class of models to capture this feature by allowing the default event of certain firms to have a ripple effect on credit spreads of other firms. In particular, we distinguish between two types of firms: primary firms and secondary firms. The latter firms may be affected by the demise of a primary firm. For example, a secondary firm may carry significant debt from a primary firm, or a significant portion of its sales may flow through a primary firm. So, when the primary firm defaults, the credit spreads of the secondary firm may jump up. Alternatively, the default of a primary firm could be good news for a secondary firm, in that it now may play a bigger role in the competitive market. In this case, an unanticipated default of its large competitor could result in a surprise downward shock to all credit spreads along the maturity spectrum of the secondary firm. Such unidirectional contagion models were first considered by Jarrow and Yu (2001) and $\mathrm{Yu}(2007)$.

The models developed here require additional parameters, some of which can only be estimated from historical data on a large cross section of firms as they relate to rare events. However, our approach yields models that produce internally consistent prices that can then be used to assess the impact of systemic shocks created by highly correlated jumps in credit spread curves across firms. As such, the main application of the models presented below is geared towards revaluing creditsensitive derivative products under different systemic risk scenarios. They provide an important tool for financial stress testing and risk management purposes.

\footnotetext{
${ }^{15}$ The limitations of the conditional independence assumption have been well-documented for reduced-form credit risk models. Examples include Hull and White (2001) and Schönbucher and Schubert (2002). Yu (2005) argues that the apparent low correlation is not a problem of the conditionally independent default approach, but rather with the choice of state variables. Specifically, a limited set of state variables or factors may not be sufficient to model the changes in intensities, and perhaps additional state variables are necessary. Using a fixed set of state variables, Das, Duffie, Kapadia, and Saita (2007) test whether default events can be modeled as conditionally independent and reject this hypothesis. Lando and Nielsen (2008), using the same sample, cannot reject this hypothesis, but, using alternative tests, they do find support for contagion effects that take place through firm covariates. Duffie, Eckner, Horel, and Saita (2008) estimate frailty models in which firms could be jointly exposed to unobservable risk factors.

${ }^{16}$ For further discussions on default clustering, contagion and the valuation of portfolio credit derivatives see Egloff, Leippold, and Vanini (2007), Kraft and Steffensen (2007), and Zheng and Jiang (2009).
} 
Assume that there are $m_{B}$ primary firms whose default could affect the credit spreads of some secondary firm $B$. The primary firms are labeled $A_{1}, \ldots, A_{m_{B}}$. Their credit spreads do not depend on the default state of other firms. As in Section 2, the state variables for primary firm $\mathrm{A}_{i}$ are $X_{A_{i}}(t)=\left\{\Phi(t), \Upsilon_{A_{i}}(t)\right\}$. For our secondary firm B, the number of state variables influencing its credit spread curve is significantly enhanced. In addition to $\Phi(t)$, we may need to know the credit spreads of all relevant surviving primary firms. Let

$$
X_{B}(t)=\left\{\Phi(t), \Upsilon_{A_{1}}(t), \ldots, \Upsilon_{A_{m_{B}}}(t), \Upsilon_{B}(t), Y(t)\right\}
$$

where $\Upsilon_{A_{i}}(t)$, is empty if firm $\mathrm{A}_{i}$ has defaulted prior to date $t$. To model a secondary firm's process, we need to know the correlation structure among all the primary firms' diffusive factors, as well as how these diffusive terms correlate with the secondary firm's diffusive component. In addition, we need to track the primary firms' default status. Given the instantaneous credit spread $\lambda_{A_{i}}(t)$ for primary firm $A_{i}$, and the risk-neutral expected fractional loss in market value at default, $\ell_{A_{i}}(t)$, we can compute its risk-neutral default intensity as $\eta_{A_{i}}(t)=\lambda_{A_{i}}(t) / \ell_{A_{i}}(t)$. It is this instantaneous probability that determines firm $A_{i}$ 's likelihood of default in the next time increment.

The risk-neutral dynamics of the forward riskless rates and credit spreads are given by (2) and

$$
\begin{aligned}
d \lambda_{A_{i}}(t, T)= & \mu_{A_{i}}(t, T) d t+\sigma_{A_{i}}(t, T) d z_{A_{i}}(t)+c_{f A_{i}}(t, T) d N_{f}(t), \forall t \leq \tau_{A_{i}}, i=1, \ldots, m_{B},(34) \\
d \lambda_{B}(t, T)= & \mu_{B}(t, T) d t+\sigma_{B}(t, T) d z_{B}(t)+c_{f B}(t, T) d N_{f}(t) \\
& +\sum_{i=1}^{m_{B}} c_{A_{i} B}(t, T)\left(1-Y_{A_{i}}(t)\right) d Y_{A_{i}}(t), \quad \forall t \leq \tau_{B}
\end{aligned}
$$

The date- 0 riskless forward curve and the date- 0 credit spread curves of firms $\mathrm{A}_{i}$ and $B$ are initialized to their observable values. The forward credit spread of firm $B$ is driven by a continues diffusive term, $d z_{B}(t)$, where $z_{B}(t)=\left(z_{B_{1}}(t), \ldots, z_{B_{n}}(t)\right)^{\prime}$ is an $n$-dimensional standard Brownian motion with $E\left(d z_{f}(t) d z_{B}^{\prime}(t)\right)=\Sigma_{m \times n}^{B} d t$, where $\left(\Sigma^{B}\right)_{i j}=\rho_{i j}^{B}$. In addition, it is correlated with firm $\mathrm{A}_{i}$ 's diffusive term according to $E\left(d z_{A_{i}}(t) d z_{B}^{\prime}(t)\right)=\Sigma_{n \times n}^{A_{i} B} d t$, where

$$
\left(\Sigma^{A_{i} B}\right)_{k l}=\rho_{k l}^{A_{i} B}
$$

Similarly, the primary firms' diffusive terms are correlated according to $E\left(d z_{A_{i}}(t) d z_{A_{j}}^{\prime}(t)\right)=$ $\Sigma_{n \times n}^{A_{i} A_{j}} d t$, where $\left(\Sigma^{A_{i} A_{j}}\right)_{k l}=\rho_{k l}^{A_{i} A_{j}}$.

When there is a jump in the riskless curve, then there is a corresponding jump in the credit spread curve of firm B. Further, default of any of the primary firms $\mathrm{A}_{i}$ could transmit shocks in the credit spreads of the secondary firm. As before, the volatility factor, $\sigma_{B}(t, T)$, is predictable, while $c_{f B}(t, T)$ and $c_{A_{i} B}(t, T)$ are deterministic functions of time to maturity, $T-t$. More specifically, we impose volatility structures $\sigma_{B}(t, T)=\left(\sigma_{B_{1}}(t, T), \ldots, \sigma_{B_{n}}(t, T)\right)$ of the form:

$$
\sigma_{B_{j}}(t, T)=h_{B_{j}}(t) e^{-\kappa_{B_{j}}(T-t)}
$$


and jump impact factors given by:

$$
c_{f B}(t, T)=c_{f B} e^{-\gamma_{f B}(T-t)}
$$

where $h_{B_{j}}(t)$ is a predictable function that depends on a set of state variables.

Proposition 3 below provides the linkage for credit spreads of a secondary firm to its date-0 credit spread curve, through a well-defined collection of state variables.

\section{Proposition 3}

Given the risk-neutral dynamics (2), (34) and (35) together with the volatility and impact structures specified in (13) through (16), (36) and (37), assuming recovery of market value and that the impact of a primary firm's default on secondary firm, $B$, is constant, $c_{A_{j} B}(t, T)=c_{A_{j} B}$, the price of a defaultable zero-coupon bond issued by firm $B$ is given by $\Pi_{B}(t, T)=V_{B}(t, T) 1_{\left\{\tau_{B}>t\right\}}$, where $V_{B}(t, T)=P(t, T) S_{B}(t, T)$ and

$$
\begin{aligned}
S_{B}(t, T)= & \frac{S_{B}(0, T)}{S_{B}(0, t)} \exp \left(-A_{0}^{B}(t, T)-\sum_{j=1}^{n}\left(K_{0, j}^{B}(t, T) \xi_{0, j}^{B}-K_{1, j}^{B}(t, T) \xi_{1, j}^{B}\right)\right) \\
& \times \exp \left(\sum_{i=1}^{m} \sum_{j=1}^{n}\left(K_{2, i j}^{B}(t, T) \xi_{2, i j}^{B}-K_{3, i j}^{B}(t, T) \xi_{3, i j}^{B}-K_{4, i j}^{B}(t, T) \xi_{4, i j}^{B}\right)-K_{5}^{B}(t, T) \xi_{5}^{B}(t)\right) \\
& \times \exp \left(\sum_{i=1}^{m_{B}}\left(\left(1-e^{-c_{A_{i} B}(T-t)}\right) U_{A_{i} B}(t)-c_{A_{i} B}(T-t) Y_{A_{i}}(t)\right)\right) .
\end{aligned}
$$

Here, $A_{0}^{B}(t, T)$, the $K^{B}$ coefficients and the dynamics of the $\xi^{B}$ state variables are all defined as in Proposition 2, but now with respect to the secondary firm B, rather than the primary firm, A, and

$$
U_{A_{i} B}(t)=\int_{0}^{t \wedge \tau_{A_{i}}} \eta_{A_{i}}(u) e^{-c_{A_{i} B}(t-u)} d u, \quad i=1, \ldots, m_{B} .
$$

Proof: See Appendix A.

Unlike primary firms, the prices of secondary firms' bonds depend on the default status of primary firms, as well as the timing of the primary firms' defaults. In addition, computing $U_{A_{i} B}(t)$ requires knowledge of firm $\mathrm{A}_{i}$ 's risk-neutral default intensity $\eta_{A_{i}}(t)$, which in turn requires knowledge of the state variables for the primary firm $\mathrm{A}_{i}$.

To highlight the computational effort involved with our models for secondary firms, consider a simple specification with two stochastic drivers, one for interest rates $(m=1)$ and one for each firm's credit spreads $(n=1)$. We show that bond prices are Markovian in at most $8+2 m_{B}$ state variables, with no more than $3+m_{B}$ stochastic drivers. Even in this simple framework, systemic credit shocks can be captured via the response of each firm's credit spreads to shocks in the economy that cause jumps in interest rates and in credit spreads, as well as ripple effects on credit spreads 
of secondary firms caused by defaults of primary firms.

\section{Corollary to Proposition 3}

For $m=n=1$, an equivalent representation for secondary firm B's bond prices, $\Pi_{B}(t, T)=$ $V_{B}(t, T) 1_{\left\{\tau_{B}>t\right\}}$, is $V_{B}(t, T)=P(t, T) S_{B}(t, T)$, where

$$
\begin{aligned}
S_{B}(t, T)= & \frac{S_{B}(0, T)}{S_{B}(0, t)} \exp \left(-A_{0}^{B}(t, T)-K\left(t, T ; \kappa_{B}\right)\left(\lambda_{B}(t)-\lambda_{B}(0, t)\right)\right) \\
& \times \exp \left(-\sum_{j=1}^{3} K_{j}^{B}(t, T) \xi_{j}^{B}(t)-K_{5}^{B}(t, T) \xi_{5}^{B}(t)\right) \\
& \times \exp \left(-(T-t) \sum_{i=1}^{m_{B}} c_{A_{i} B} Y_{A_{i}}(t)+K\left(t, T ; \kappa_{B}\right) \sum_{i=1}^{m_{B}} c_{A_{i} B} Y_{A_{i}}(t)\right) \\
& \times \exp \left(-\sum_{i=1}^{m_{B}}\left[K\left(t, T ; \kappa_{B}\right)-\frac{1}{c_{A_{i} B}}\left(1-e^{-c_{A_{i} B}(T-t)}\right)\right] c_{A_{i} B} U_{A_{i} B}(t)\right) .
\end{aligned}
$$

Proof: See Appendix A.

Notice that if exogenous jumps in instantaneous riskless rates and credit spreads are turned off, and if default of a primary firm does not induce any contagion effects, then this Corollary is reduced to the Corollary to Proposition 2.

\subsection{The Importance of Default Contagion}

In this section, we implement our model to price an array of multi-name credit-sensitive products. Our primary goals are to highlight the importance of building contagion effects into our model structure, and to illustrate that these models can be used in larger applications involving multiple names. In our first example, we price the counterparty risk in a default insurance contract. In our second application, we price tranches of synthetic CDS indices, effectively extending the two-firm setting of the first example to a much larger portfolio of credits.

\subsubsection{Counterparty Risk in Insurance Contracts}

Revisiting the CCDS example from Section 3.2.2, we now explicitly take into account the default risk of the protection seller (firm A), and compute the price of the counterparty risk in the insurance contract itself. This source of counterparty risk borne by the protection buyer, firm F, should not be confused with the counterparty risk in the underlying interest-rate swap contract. Instead, it is the risk associated with default of the protection seller in the insurance contract, firm A, prior to default of the counterparty in the underlying interest-rate swap contract, firm B.

To better reflect reality, we assume that the protection seller, A, is a primary firm of good 
credit quality, and that the counterparty to the underlying swap contract, B, is a riskier secondary firm. We first compute the at-market CCDS rate when the default risk of the protection seller is ignored, and then the reduction in at market rates when the default risk of firm $\mathrm{A}$ is taken into account. ${ }^{17}$ Figure 4 reports how the reduction in at-market CCDS rates due to the default risk of the protection seller, expressed as a fraction of the at-market CCDS rates when the default risk of the protection seller is ignored, varies as key correlation parameters change. ${ }^{18}$ The correlation parameters under investigation are $\rho^{A}$ and $\rho^{B}, \rho^{A B}, c_{A B}$ and $\eta_{f}$. For the latter two, it is important to emphasize that the initial credit spread curves are kept fixed for each scenario to ensure that the focus is on correlation effects, and not contaminated by changes to the level of the initial term structure of credit spreads.

\section{Figure 4 Here}

In the top left panel, we find a significant negative relationship between the prices of insurance against default of the protection seller in the CCDS contract and diffusive correlations between interest rates and credit spreads, as measured by $\rho^{A}$ and $\rho^{B}$. This is in line with the results from Section 3.2. Moreover, we find a positive impact of the parameter $\rho^{A B}$, which captures the diffusive correlation between credit spreads of firms A and B, on the difference between at-market CCDS rates when the default risk of the protection seller is ignored and when it is priced. A positive correlation between the protection seller in the CCDS contract, A, and the counterparty to the underlying swap contract, B, implies that at times when firm A's default risk is high (and hence the protection seller in the CCDS contract is likely to default), default risk associated with the underlying swap contract is also high. Conversely, if the correlation is negative, then at times when firm A's default risk is high, default risk associated with the underlying swap contract is low. As a result, the reduction in CCDS rates due to the default risk of the protection seller increases as $\rho^{A B}$ increases.

When compared to the diffusive correlation parameters, the results for the credit spread impact factor, $c_{A B}$, which measures the impact of default of the protection seller $\mathrm{A}$ on the credit spread curve of firm $\mathrm{B}$, are striking. If $c_{A B}$ is increased from zero to 0.1 , the reduction in CCDS rates due to the default risk of the protection seller, expressed as a fraction of the CCDS rate when the default risk of the protection seller is ignored, increases from two to over five percent. An increase of the instantaneous credit spread by 1,000 basis points translates into an increase of almost twenty basis points in the likelihood of default over the next week. The appropriate size of $c_{A B}$ ultimately

\footnotetext{
${ }^{17}$ The former is computed as in Section 3.2.2, but under the assumption that the interest-rate swap counterparty is a secondary firm. To compute CCDS rates that take into account the default risk of the protection seller, we set the value of the protection leg in equation (33) to zero if A defaults prior to B.

${ }^{18} \mathrm{~A}$ benchmark set of parameter values is given by $\kappa_{f}=\kappa_{A}=\kappa_{B}=0.1, \tilde{h}_{f}(t)=r(t), \tilde{h}_{A}(t)=0.5 \lambda_{A}(t)$, $\tilde{h}_{B}(t)=0.5 \lambda_{B}(t)$, and $\bar{h}_{f}=\bar{h}_{A}=\bar{h}_{B}=10^{6}$. When jumps in riskless rates are permitted, they have a dampening effect on rates as a function of maturity, with $\gamma_{f}=\gamma_{f A}=\gamma_{f B}=0.5$. We set the jump size parameter $c_{f}$ equal to -0.01. When $\rho^{A}$ and $\rho^{B}$ are away from zero, we set $\rho^{A B}$ equal to their product. We assume there is no recovery at default, and that the initial yield curve is flat at $4 \%$. Our Monte Carlo simulations use weekly time steps, and 10,000 sample paths with antithetic variates.
} 
depends on the closeness of the relationship between the primary firm A and the secondary firm B. For example, $c_{A B}$ is likely to be higher if both firms belong to the same sector, and lower if they are close competitors. The final figure shows the impact of $\eta_{f}$. Although increasing the jump intensity parameter has a positive effect on the reduction in CCDS rates due to the default risk of the protection seller, the impact is of moderate size when compared to that of $c_{A B}$.

The relative importance of the credit spread impact factor $c_{A B}$ increases as the volatility of credit spreads decreases. When credit spread volatility is low, the impact of the diffusive correlation between interest rates and credit spreads, $\rho^{A}$ and $\rho^{B}$, and of the diffusive correlation of credit spreads across firms, $\rho^{A B}$, is significantly smaller. This highlights the importance of including credit spread impact factors in our models.

\subsubsection{CDS Index Tranches}

To further emphasize the effectiveness of the credit spread impact factor $c_{A B}$ in generating a wide range of prices for multi-name credit derivatives, we extend the two-firm setting of the previous section to a much larger portfolio of credits. ${ }^{19}$ Specifically, we now investigative the sensitivity of CDS index tranche prices to $c_{A B}$. We mimic the setup of the benchmark 5-year high-yield CDS index (ticker CDX.NA.HY, for details see www.markit.com) by considering a synthetic portfolio of 100 5-year speculative-grade CDS contracts, and price tranches on its loss distribution. CDX.NA.HY indices are sliced into five tranches: the equity tranche that incurs the first $0-10 \%$ of losses, junior mezzanine and senior mezzanine tranches that are responsible for the subsequent $10-15 \%$ and 15 $25 \%$ of losses, and a senior and super-senior tranche that account for the next $25-35 \%$ and $35-100 \%$ of losses, respectively.

Figure 5 shows simulated tranche prices as a function of $c_{A B}$, while holding everything else, including the initial credit spread curves of the underlying names, fixed. We assume that there are five primary firms and 95 secondary firms in the index. As the credit spread impact factor increases from 0 to 0.1 , senior tranche prices increase from less than 100 basis point to almost 600 basis points, due to an increase in the likelihood of a large number of defaults occurring within a relatively short time span. At the same time, the value of the junior mezzanine tranche decreases from $33.4 \%$ (at $c_{A B}=0$ ) to $17.9 \%$ (at $c_{A B}=0.1$ ). This decrease in value is due to the fact that an increase in default contagion, while keeping the level of initial credit spread curves constant, indirectly increases the likelihood of only a few firms defaulting, the only scenario under which junior mezzanine tranche investors do not have to pay.

\section{Figure 5 Here}

\footnotetext{
${ }^{19}$ An alternative to modeling CDS index tranches based on the individual credit spread curves is proposed in Longstaff and Rajan (2008). The authors argue that the distribution of total portfolio losses represents a sufficient statistic for valuing tranches, and assume that the dynamics of total portfolio losses are driven by three independent Poisson processes that capture firm-specific, industry-wide and market-wide default events, respectively. Is is important to note that such a structure could easily be implemented using a straight-forward extension of the model in Section 2 to include two additional jump processes.
} 
In summary, our results highlight the importance of including credit spread impact factors in our models that permit jumps to occur in the intensities of secondary bonds when a primary bond defaults. The senior tranche investor in a CDS index has to make payments only if a relatively large number of defaults occurs within a rather short time frame. Similarly, the protection seller in the CCDS contract fails to make a promised payment only in the scenario where it defaults before the counterparty in the underlying swap contract, that is, in cases where the default of firm A is followed within a short time period by a default of firm B. To further emphasize the effectiveness of the credit spread impact factor $c_{A B}$ in generating such default clustering, Figure 6 shows sample paths for the timing of default events over a 5-year period. Each sample path is associated with a particular value for $c_{A B}$, while everything else is kept the same. The plots indicate that for higher credit spread impact factors, defaults are more likely to occur in clusters, and that they are spread out more evenly across time if no feedback effects from defaults of primary firms are allowed.

Figure 6 Here

\subsection{The Importance of the Initial Credit Spread Curve Distribution}

For the correlation studies above, it has been crucial to keep initial credit spread curves fixed. But since an appealing feature of our family of models is that they fully incorporate the current credit spread curve information, we investigate how sensitive multi-name products are to the distribution, across firms, of initial credit spread curves. Our goal is to demonstrate the importance of taking into account the full credit spread curve information for each firm, and their distribution across firms. The latter should be of particular concern when pricing basket credit derivatives.

Continuing with our CDS index example from Section 4.1.2, Table 4 shows the simulated tranche prices of a 5-year CDX.NA.HY index for different distributions of initial credit spread curves. In particular, we consider two simplified scenarios. First, we investigate the case where the initial credit spread curve is flat for all firms, but possibly at different levels. The credit spread level for each firm is simulated from a uniform distribution that is centered around 5\%, say. Second, we simulate tranche prices under the assumption that the initial credit spread curve is a linear function of time, with a fixed point of $5 \%$ at 2.5 years (half the contract term).

Table 4 Here

Our results indicate that the mezzanine and senior tranche prices increase significantly as the probability of higher initial forward credit spreads at the short end of the term structure increases. In the first example, the junior mezzanine (senior) tranche price increases by $2 \%$ (9\%) when moving from a flat initial credit spread curve at $5 \%$ for all firms to a flat initial credit spread curve at a level that is uniformly distributed, across firms, between 0 and $10 \%$.

The effect of changes in the slope of the initial credit spread curves is even more dramatic. For our second set of distributions, the results displayed in Table 4 show that the junior mezzanine 
(senior) tranche price increases by $43 \%(27 \%)$ as the initial spread curve, for each firm, is tilted from being flat at $5 \%$ to being downward sloping from $8.75 \%$ (at 0-year maturity) to $1.25 \%$ (at 5 -year maturity). Junior mezzanine tranche prices decrease dramatically as initial spread curves are tilted from being flat to being upward sloping, whereas senior tranche prices remain fairly flat.

\section{Conclusion}

Our paper's contribution has been to establish a family of multifactor HJM term-structure models for valuing interest- and credit-sensitive cash flows that permit an exponential affine representation of riskless and risky bond prices while offering significant flexibility in the choice of volatility structures. We accomplish this without restricting our processes to the class of affine jump diffusions (Duffie and Kan (1996), Duffie, Pan, and Singleton (2000)). Rather, we only need to curtail the forward rate volatilities in relation to their spot rate volatility counterparts. Our class of resulting volatility structures complements the Duffie-Kan family by allowing a broad choice of specifications while still retaining an exponential affine representation.

To facilitate empirical studies, we provide an illustrative example of how estimation can proceed using an extended Kalman filter. We show that, from a practical perspective, estimating models in our family is typically no more difficult than estimating models in the workhorse affine family. For cases where the affine models require solving systems of Riccati equations, estimating models with the same number of stochastic drivers in our family may even be easier. It remains for future empirical research to establish models within our family that best describe the joint dynamics of interest rates and credit spreads.

This paper also considers how failures of certain firms could affect the credit spread curves of other surviving firms. The complexity of such contagion models increases since the dimensionality of the system of equations needed to characterize the economy expands. The applications considered involve pricing multiple sources of counterparty credit risk in derivative transactions and credit derivatives written on multiple names, both of which would not be feasible with general HJM models. Interestingly, our models incorporate more information than most in pricing CDS index tranches and allow us to explore more precisely the impact of heterogeneity in the index composition.

Our family of models captures systemic credit shocks through common jumps and, more importantly, default contagion. The primary role of incorporating the latter into our models is to permit stress tests to be conducted on a large portfolio of credits. By revaluing derivative products like CDS index tranches under different assumptions on credit spread impact factors, we can control the degree of clustering of defaults. Moreover, our valuations under differing degrees of possible impact factors are all conducted in a consistent no-arbitrage framework. As the recent financial crisis reminded us, credit risks are not easily diversified, and the role of stress testing credit-sensitive portfolios cannot be understated. 


\section{Appendices}

\section{A Proofs}

\section{Proof of Proposition 1}

Under the risk-neutral measure, the expected instantaneous return of the riskless bond should equal the riskless rate, $r(t)$. Absence of arbitrage therefore requires the expected instantaneous return in equation (3) to be equal to $r(t) d t$. Hence:

$$
r(t)+\frac{1}{2} \sigma_{p}(t, T) \sigma_{p}^{\prime}(t, T)-\int_{t}^{T} \mu_{f}(t, u) d u+\left(e^{-K_{p}(t, T)}-1\right) \eta_{f}=r(t)
$$

from which:

$$
\int_{t}^{T} \mu_{f}(t, u) d u=\frac{1}{2} \sigma_{p}(t, T) \sigma_{p}^{\prime}(t, T)+\left(e^{-K_{p}(t, T)}-1\right) \eta_{f} .
$$

Differentiating with respect to $T$, we obtain:

$$
\mu_{f}(t, T)=\sigma_{p}(t, T) \sigma_{f}^{\prime}(t, T)-c_{f}(t, T) e^{-K_{p}(t, T)} \eta_{f}
$$

This restriction on the drift term is the classic Heath-Jarrow-Morton restriction for forward rates under the risk-neutral measure when the dynamics follow a jump diffusion as in (2).

Now turn to the risky bond. Under our assumptions, the dynamics for $V_{A}(t, T)$ are:

$\frac{d V_{A}(t, T)}{V_{A}(t, T)}=\mu_{V_{A}}(t, T) d t-\sigma_{p}(t, T) d z_{f}(t)-\sigma_{S_{A}}(t, T) d z_{A}(t)+\left(e^{-K_{p}(t, T)-K_{f A}(t, T)}-1\right) d N_{f}(t)$

where

$$
\begin{aligned}
\mu_{V_{A}}(t, T)= & \lambda_{A}(t)-\int_{t}^{T} \mu_{A}(t, u) d u+\frac{1}{2} \sigma_{S_{A}}(t, T) \sigma_{S_{A}}^{\prime}(t, T)+r(t)-\left(e^{-K_{p}(t, T)}-1\right) \eta_{f} \\
& +\sigma_{p}(t, T) \Sigma^{A} \sigma_{S_{A}}^{\prime}(t, T) .
\end{aligned}
$$

If we assume that at the time of default the recovery value is proportional to the market value of the bond just prior to default, absence of arbitrage implies that we can price the risky bond as the risk-neutral expectation of discounted cash flows. The discount rate is modified from $r$ to $r+\lambda_{A}$ to reflect default and recovery risk (see Duffie and Singleton (1999a)). Given that

$$
\begin{aligned}
E\left(\frac{d V_{A}(t, T)}{V_{A}(t, T)}\right) & =\left(r(t)+\lambda_{A}(t)\right) d t \\
& =\left(\mu_{V_{A}}(t, T)+\left(e^{-K_{p}(t, T)-K_{f A}(t, T)}-1\right) \eta_{f}\right) d t
\end{aligned}
$$


we have:

$$
\begin{aligned}
\int_{t}^{T} \mu_{A}(t, u) d u= & \frac{1}{2} \sigma_{S_{A}}(t, T) \sigma_{S_{A}}^{\prime}(t, T)+\sigma_{p}(t, T) \Sigma^{A} \sigma_{S_{A}}^{\prime}(t, T) \\
& +\left(e^{-K_{p}(t, T)-K_{f A}(t, T)}-e^{-K_{p}(t, T)}\right) \eta_{f} .
\end{aligned}
$$

Differentiating this expression with respect to $T$ yields the result.

\section{Proof of Proposition 2}

(i) First consider the riskless bond. Substituting the volatility and impact expressions into equation (10), and using (2), leads upon simplification to:

$$
\begin{aligned}
f(t, T)= & f(0, T)+\sum_{i=1}^{m} \frac{1}{\kappa_{f_{i}}} e^{-\kappa_{f_{i}}(T-t)}\left(\psi_{1, i}(t)-e^{-\kappa_{f_{i}}(T-t)} \psi_{2, i}(t)\right)+c_{f} e^{-\gamma_{f}(T-t)} \psi_{3}(t) \\
& -c_{f} \eta_{f} L_{f}(t, T),
\end{aligned}
$$

where, for $i=1, \ldots, m$,

$$
\begin{aligned}
\psi_{1, i}(t) & =\int_{0}^{t} h_{f_{i}}^{2}(u) e^{-\kappa_{f_{i}}(t-u)} d u+\kappa_{f_{i}} \int_{0}^{t} h_{f_{i}}(u) e^{-\kappa_{f_{i}}(t-u)} d z_{f_{i}}(u), \\
\psi_{2, i}(t) & =\int_{0}^{t} h_{f_{i}}^{2}(u) e^{-2 \kappa_{f_{i}}(t-u)} d u \\
\psi_{3}(t) & =\int_{0}^{t} e^{-\gamma_{f}(t-u)} d N_{f}(u)
\end{aligned}
$$

and

$$
\begin{aligned}
L_{f}(t, T) & =\int_{0}^{t} e^{-\gamma_{f}(T-u)-\frac{c_{f}}{\gamma_{f}}\left(1-e^{-\gamma_{f}(T-u)}\right)} d u \\
& =\frac{1}{c_{f}}\left(e^{-\frac{c_{f}}{\gamma_{f}}\left(1-e^{-\gamma_{f}(T-t)}\right)}-e^{-\frac{c_{f}}{\gamma_{f}}\left(1-e^{-\gamma_{f} T}\right)}\right) .
\end{aligned}
$$

The result follows by substituting equation (A.2) into (1), and simplifying.

(ii) The forward credit spread, $\lambda_{A}(t, T)$, can be expressed as:

$$
\lambda_{A}(t, T)=\lambda_{A}(0, T)+\int_{0}^{t} \mu_{A}(u, T) d u+\int_{0}^{t} \sigma_{A}(u, T) d z_{A}(u)+\int_{0}^{t} c_{f A}(u, T) d N_{f}(u) .
$$

Substituting the volatility and jump impact factor restrictions given in equations (13) through (16) into the drift restriction from Proposition 1, and using the above equation, we obtain upon simpli- 
fication:

$$
\begin{aligned}
\lambda_{A}(t, T)= & \lambda_{A}(0, T)+G_{A}(t, T)+\sum_{j=1}^{n} \frac{1}{\kappa_{A_{j}}} e^{-\kappa_{A_{j}}(T-t)} \xi_{0, j}(t)-\sum_{j=1}^{n} \frac{1}{\kappa_{A_{j}}} e^{-2 \kappa_{A_{j}}(T-t)} \xi_{1, j}(t) \\
& -\sum_{i=1}^{m} \sum_{j=1}^{n} \rho_{i j}^{A}\left(\frac{1}{\kappa_{f_{i}}}+\frac{1}{\kappa_{A_{j}}}\right) e^{-\left(\kappa_{f_{i}}+\kappa_{A_{j}}\right)(T-t)} \xi_{2, i j}(t)+\sum_{i=1}^{m} \sum_{j=1}^{n} \frac{\rho_{i j}^{A}}{\kappa_{A_{j}}} e^{-\kappa_{f_{i}}(T-t)} \xi_{3, i j}(t) \\
& +\sum_{i=1}^{m} \sum_{j=1}^{n} \frac{\rho_{i j}^{A}}{\kappa_{f_{i}}} e^{-\kappa_{A_{j}}(T-t)} \xi_{4, i j}(t)+c_{f A} e^{-\gamma_{f A}(T-t)} \xi_{5}(t)
\end{aligned}
$$

where $G_{A}(t, T)=\int_{0}^{t} g_{A}(u, T) d u$. Note that if $\gamma_{f A}=\gamma_{f}$, analytic solutions are available for $G_{A}(t, T)$. For $i=1, \ldots, m$ and $j=1, \ldots, n$,

$$
\begin{aligned}
\xi_{0, j}(t) & =\int_{0}^{t} e^{-\kappa_{A_{j}}(t-u)} h_{A_{j}}^{2}(u) d u+\kappa_{A_{j}} \int_{0}^{t} e^{-\kappa_{A_{j}}(t-u)} h_{A_{j}}(u) d z_{A_{j}}(u), \\
\xi_{1, j}(t) & =\int_{0}^{t} e^{-2 \kappa_{A_{j}}(t-u)} h_{A_{j}}^{2}(u) d u \\
\xi_{2, i j}(t) & =\int_{0}^{t} e^{-\left(\kappa_{f_{i}}+\kappa_{A_{j}}\right)(t-u)} h_{f_{i}}(u) h_{A_{j}}(u) d u \\
\xi_{3, i j}(t) & =\int_{0}^{t} e^{-\kappa_{f_{i}}(t-u)} h_{f_{i}}(u) h_{A_{j}}(u) d u \\
\xi_{4, i j}(t) & =\int_{0}^{t} e^{-\kappa_{A_{j}}(t-u)} h_{f_{i}}(u) h_{A_{j}}(u) d u \\
\xi_{5}(t) & =\int_{0}^{t} e^{-\gamma_{f A}(t-u)} d N_{f}(u) .
\end{aligned}
$$

The result then follows by substituting equation (A.3) into (6), and simplifying.

\section{Proof of Corollary to Proposition 2}

From equation (A.2), consider the case $m=1$ with no jumps, and put $T=t$ to obtain:

$$
r(t)=f(0, t)+\frac{1}{\kappa_{f}}\left(\psi_{1}(t)-\psi_{2}(t)\right)
$$

where second subscripts on the $\psi$ variables have been dropped. From this, an expression for $\psi_{1}(t)$ can be obtained. Substituting it back into equation (A.2) yields:

$$
f(t, T)=f(0, T)+e^{-\kappa_{f}(T-t)}(r(t)-f(0, t))+\frac{1}{\kappa_{f}}\left(e^{-\kappa_{f}(T-t)}-e^{-2 \kappa_{f}(T-t)}\right) \psi_{2}(t),
$$

from which the riskless bond price formula follows. The dynamics of $r(t)$ follow using the equation:

$$
d r(t)=d f(t, t)+\left.\frac{d}{d t} f(u, t)\right|_{u \rightarrow t} .
$$

To compute the risky bond price, we use equation (A.3) for the case $n=1$ and no jumps. 
Setting $T=t$, this allows us to express $\xi_{0,1}(t)$ in terms of $\lambda_{A}(t)$ and the other state variables. Substituting the resulting expression back into equation (A.3), dropping unnecessary subscripts and rearranging terms leads to:

$$
\begin{aligned}
\lambda_{A}(t, T)= & \lambda_{A}(0, T)+e^{-\kappa_{A}(T-t)}\left(\lambda_{A}(t)-\lambda_{A}(0, t)\right)+\frac{1}{\kappa_{A}} e^{-\kappa_{A}(T-t)}\left(1-e^{-\kappa_{A}(T-t)}\right) \xi_{1}(t) \\
& +\rho^{A}\left(\frac{1}{\kappa_{f}}+\frac{1}{\kappa_{A}}\right) e^{-\kappa_{A}(T-t)}\left(1-e^{-\kappa_{f}(T-t)}\right) \xi_{2}(t) \\
& +\frac{\rho^{A}}{\kappa_{A}}\left(e^{-\kappa_{f}(T-t)}-e^{-\kappa_{A}(T-t)}\right) \xi_{3}(t) .
\end{aligned}
$$

Note that the $\xi_{4}(t)$ terms cancel out, effectively reducing the number of state variables that describe credit spreads from five in Proposition 2 to four. $S_{A}(t, T)$ then follows by integrating the above equation, which allows us to compute the risky bond price $\Pi_{A}(t, T)=P(t, T) S_{A}(t, T) 1_{\left\{\tau_{A}>t\right\}}$. The dynamics of the path statistics follow from the main proposition, while the dynamics of $\lambda_{A}(t)$ follow from:

$$
d \lambda_{A}(t)=d \lambda_{A}(t, t)+\left.\frac{d}{d t} \lambda_{A}(u, t)\right|_{u \rightarrow t}
$$

\section{Proof of Proposition 3}

Under our assumptions, the dynamics for $V_{B}(t, T)$ are:

$$
\begin{aligned}
\frac{d V_{B}(t, T)}{V_{B}(t, T)}= & \mu_{V_{B}}(t, T) d t-\sigma_{p}(t, T) d z_{f}(t)-\sigma_{S_{B}}(t, T) d z_{B}(t)+\left(e^{-K_{p}(t, T)-K_{f B}(t, T)}-1\right) d N_{f}(t) \\
& +\sum_{i=1}^{m_{B}}\left(e^{-K_{A_{i} B}(t, T)}-1\right)\left(1-Y_{A_{i}}(t)\right) d Y_{A_{i}}(t)
\end{aligned}
$$

where $\sigma_{S_{B}}(t, T)$ and $K_{f B}(t, T)$ are defined analogous to (8) and (9), $K_{A_{i} B}(t, T)=\int_{t}^{T} c_{A_{i} B}(t, u) d u$, and $\mu_{V_{B}}(t, T)$ is given as in (A.1), with A replaced by B.

If we assume fractional loss of market value at default, absence of arbitrage implies that the instantaneous risk-neutral expected return on firm B's bond is $\left(r(t)+\lambda_{B}(t)\right) d t$. Substituting this constraint into the expression for $\mu_{V_{B}}(t, T)$, and following the same steps as for primary bonds in the Proof to Proposition 1, yields:

$$
\begin{aligned}
\mu_{B}(t, T)= & \sigma_{S_{B}}(t, T) \sigma_{B}^{\prime}(t, T)+\sigma_{f}(t, T) \Sigma^{B} \sigma_{S_{B}}^{\prime}(t, T)+\sigma_{p}(t, T) \Sigma^{B} \sigma_{B}(t, T)+g_{B}(t, T) \\
& -\sum_{i=1}^{m_{B}} \eta_{A_{i}}(t) c_{A_{i} B}(t, T) e^{-K_{A_{i} B}(t, T)}\left(1-Y_{A_{i}}(t)\right)
\end{aligned}
$$

where $g_{B}(t, T)=\eta_{f}\left(c_{f}(t, T) e^{-K_{p}(t, T)}-\left(c_{f}(t, T)+c_{f B}(t, T)\right) e^{-\left(K_{p}(t, T)+K_{f B}(t, T)\right)}\right)$.

Following the same logic as used to develop the price of a primary bond in Proposition 2, the 
date- $t$ credit spread of a secondary firm is linked to its date- 0 value by:

$$
\begin{aligned}
\lambda_{B}(t, T)= & \lambda_{B}(0, T)+\int_{0}^{t} \mu_{B}(u, T) d u+\int_{0}^{t} \sigma_{B}(u, T) d z_{B}(u)+\int_{0}^{t} c_{f B}(u, T) d N_{f}(u) \\
& +\sum_{i=1}^{m_{B}} \int_{0}^{t} c_{A_{i} B}(u, T)\left(1-Y_{A_{i}}(u)\right) d Y_{A_{i}}(u) .
\end{aligned}
$$

With the assumption that the impact of a primary firm's default on firm B is constant across maturities, the last term in equation (A.6) simplifies to $\sum_{i=1}^{m_{B}} \eta_{A_{i}}(t) c_{A_{i} B} e^{-c_{A_{i} B}(T-t)}\left(1-Y_{A_{i}}(t)\right)$, and the last term in (A.7) can be written as $\sum_{i=1}^{m_{B}} c_{A_{i} B} Y_{A_{i}}(t)$.

The proof now follows by substituting the volatility and jump impact factor restrictions in (36) and (37) into the drift expression, and using the simplifications obtained from assuming that $c_{A_{i} B}(t, T)$ is constant. We obtain:

$$
\begin{aligned}
\lambda_{B}(t, T)= & \lambda_{B}(0, T)+G_{B}(t, T)+\sum_{j=1}^{n} \frac{1}{\kappa_{B_{j}}} e^{-\kappa_{B_{j}}(T-t)} \xi_{0, j}^{B}(t)-\sum_{j=1}^{n} \frac{1}{\kappa_{B_{j}}} e^{-2 \kappa_{B_{j}}(T-t)} \xi_{1, j}^{B}(t) \\
& -\sum_{i=1}^{m} \sum_{j=1}^{n} \rho_{i j}^{B}\left(\frac{1}{\kappa_{f_{i}}}+\frac{1}{\kappa_{B_{j}}}\right) e^{-\left(\kappa_{f_{i}}+\kappa_{B_{j}}\right)(T-t)} \xi_{2, i j}^{B}(t)+\sum_{i=1}^{m} \sum_{j=1}^{n} \frac{\rho_{i j}^{B}}{\kappa_{B_{j}}} e^{-\kappa_{f_{i}}(T-t)} \xi_{3, i j}^{B}(t) \\
& +\sum_{i=1}^{m} \sum_{j=1}^{n} \frac{\rho_{i j}^{B}}{\kappa_{f_{i}}} e^{-\kappa_{B_{j}}(T-t)} \xi_{4, i j}^{B}(t)+c_{f B} e^{-\gamma_{f B}(T-t)} \xi_{5}^{B}(t) \\
& +\sum_{i=1}^{m_{B}} c_{A_{i} B} Y_{A_{i}}(t)-\sum_{i=1}^{m_{B}} c_{A_{i} B} e^{-c_{A_{i} B}(T-t)} U_{A_{i} B}(t),
\end{aligned}
$$

where $G_{B}(t, T)=\int_{0}^{t} g_{B}(u, T) d u$. The $\xi^{B}$ state variables are all defined as in the Proof of Proposition 2 , but now with respect to the secondary firm $B$, rather than the primary firm, A. The result then follows by substituting the above equation into equation (6), and simplifying.

\section{Proof of Corollary to Proposition 3}

With $m=n=1$, setting $T=t$ in (A.8) and dropping unnecessary subscripts yields the instantaneous credit spread at date $t$ :

$$
\begin{aligned}
\lambda_{B}(t)= & \lambda_{B}(0, t)+G_{B}(t, t)+\frac{1}{\kappa_{B}}\left(\xi_{0}^{B}(t)-\xi_{1}^{B}(t)\right)-\rho^{B}\left(\frac{1}{\kappa_{f}}+\frac{1}{\kappa_{B}}\right) \xi_{2}^{B}(t) \\
& +\frac{\rho^{B}}{\kappa_{B}} \xi_{3}^{B}(t)+\frac{\rho^{B}}{\kappa_{f}} \xi_{4}^{B}(t)+c_{f B} \xi_{5}^{B}(t)+\sum_{i=1}^{m_{B}} c_{A_{i} B}\left(Y_{A_{i}}(t)-U_{A_{i} B}(t)\right) .
\end{aligned}
$$

Using the above expression, we can represent $\xi_{0}^{B}(t)$ in terms of the other variables. Substituting 
the expression into equation (A.8), and rearranging, leads to:

$$
\begin{aligned}
\lambda_{B}(t, T)= & \lambda_{B}(0, T)+G_{B}(t, T)+e^{-\kappa_{B}(T-t)}\left(\lambda_{B}(t)-\lambda_{B}(0, t)-G_{B}(t, t)\right) \\
& +\frac{1}{\kappa_{B}} e^{-\kappa_{B}(T-t)}\left(1-e^{-\kappa_{B}(T-t)}\right) \xi_{1}^{B}(t)+\rho^{B}\left(\frac{1}{\kappa_{f}}+\frac{1}{\kappa_{B}}\right) e^{-\kappa_{B}(T-t)}\left(1-e^{-\kappa_{f}(T-t)}\right) \xi_{2}^{B}(t) \\
& +\frac{\rho^{B}}{\kappa_{B}}\left(e^{-\kappa_{f}(T-t)}-e^{-\kappa_{B}(T-t)}\right) \xi_{3}^{B}(t)+c_{f B}\left(e^{-\gamma_{f B}(T-t)}-e^{-\kappa_{B}(T-t)}\right) \xi_{5}^{B}(t) \\
& +\sum_{i=1}^{m_{B}} c_{A_{i} B}\left(1-e^{-\kappa_{B}(T-t)}\right) Y_{A_{i}}(t)+\sum_{i=1}^{m_{B}} c_{A_{i} B}\left(e^{-\kappa_{B}(T-t)}-e^{-c A_{i} B(T-t)}\right) U_{A_{i} B}(t) .
\end{aligned}
$$

The valuation formula for risky debt issued by a secondary firm then follows directly by substituting the credit spread and forward rate expressions into equation (A.9), and simplifying.

\section{B The Extended Kalman Filter}

The standard Kalman filter recursion for the system (31)-(32) of measurement and transition equations begins with a candidate parameter vector $\Theta$. It is used to compute the unconditional expectation and covariance matrix for $X$, which we denote by $X(0 \mid 0)$ and $P(0 \mid 0)$. Simplifying notation when possible, the steps in the recursion are:

1. Use $X(t \mid t)$ and $\Theta$ to evaluate $F_{0}(t), F_{1}(t)$ and $Q(t)$.

2. Compute the one-period-ahead prediction and variance of $X(t+\Delta t)$ :

$$
X(t+\Delta t \mid t)=F_{0}(t)+F_{1}(t) X(t \mid t) \text { and } P(t+\Delta t \mid t)=F_{1}(t) P(t \mid t) F_{1}(t)^{\prime}+Q(t) .
$$

3. Compute the one-period-ahead prediction and variance of $y(t+\Delta t)$ :

$$
y(t+\Delta t \mid t)=M_{0}+M_{1}^{\prime} X(t+\Delta t \mid t) \text { and } V(t+1 \mid t)=M_{1}^{\prime} P(t+1 \mid t) M_{1}+R .
$$

4. Compute the forecast error in $y(t+\Delta t): e(t+1)=y(t+1)-y(t+1 \mid t)$.

5. Update the predictions: $X(t+\Delta t \mid t+\Delta t)=X(t+\Delta t \mid t)+P(t+\Delta t \mid t) M_{1} V^{-1}(t+\Delta t \mid t) e(t+\Delta t)$ and $P(t+\Delta t \mid t+\Delta t)=P(t+\Delta t \mid t)-P(t+\Delta t \mid t) M_{1} V^{-1}(t+\Delta t \mid t) M_{1}^{\prime} P(t+\Delta t \mid t)$.

The estimated parameter vector $\hat{\Theta}$ solves:

$$
\hat{\Theta}=\operatorname{argmax}_{\Theta} \sum_{t=1}^{T} f\left(e_{t}, V(t \mid t-\Delta t)\right),
$$

where $m_{y}$ is the size of $y(t)$ and

$$
f\left(e_{t}, V(t \mid t-\Delta t)\right)=-0.5\left(m_{y} \log (2 \pi)+\log |V(t \mid t-\Delta t)|+e(t)^{\prime} V^{-1}(t \mid t-\Delta t) e(t)\right) .
$$

In applying the Kalman filter, there are two well known approximations that can cause the resulting estimates to be inconsistent. The first is the discretization mechanism for the continuous-time 
dynamics, and the second is the linearization of these dynamics over each time increment. These approximations are typically of limited importance in practice. Indeed, extended Kalman filtering typically produces very good estimates. For a more complete discussion on this issue and the implementation of extended Kalman filters, see Duffee and Stanton (2002) and the references therein.

For the example in Section 3.1, the dynamics of the state variables,

$$
X(t)=\left(r(t), \lambda_{A}(t), \psi(t), \xi_{1}(t), \xi_{2}(t) \xi_{3}(t)\right)^{\prime}
$$

under the data-generating measure can be expressed as:

$$
d X(t)=\mu_{X}(t) d t+\sigma_{X}(t) d w(t)
$$

where $d w(t)=\left(d w_{f}(t), d \tilde{w}_{A}(t), 0_{1 \times 4}\right)^{\prime}$ and $\left(w_{f}(t), \tilde{w}_{A}(t)\right)^{\prime}$ is a two-dimensional standard Brownian motion with respect to the data-generating measure. We have

$$
\mu_{X}(t)=\left(\begin{array}{c}
\mu_{r}(t)+\nu_{f}(t) h_{f}(t) \\
\mu_{A}(t)+\nu_{f}(t) \rho^{A} h_{A}(t)+\nu_{A}(t) \sqrt{1-\rho^{A^{2}}} h_{A}((t) \\
h_{f}^{2}(t)-2 \kappa_{f} \psi(t) \\
h_{A}^{2}(t)-2 \kappa_{A} \xi_{1}(t) \\
h_{f}(t) h_{A}(t)-\left(\kappa_{f}+\kappa_{A}\right) \xi_{2}(t) \\
h_{f}(t) h_{A}(t)-\kappa_{f} \xi_{3}(t),
\end{array}\right)
$$

and

$$
\sigma_{X}(t)=\left(\begin{array}{cc}
\bar{\sigma}_{X}(t) & 0_{2 \times 4} \\
0_{4 \times 2} & 0_{4 \times 4}
\end{array}\right)
$$

where

$$
\bar{\sigma}_{X}(t)=\left(\begin{array}{cc}
h_{f}(t) & 0 \\
\rho^{A} h_{A}(t) & \sqrt{1-\rho^{A^{2}}} h_{A}(t)
\end{array}\right) .
$$

According to the transition equation (32), a closed-form expression for the discrete-time dynamics of $X(t)$ is required. In cases where such expressions are not directly available, we take a linearization of the dynamics described in (B.1) through (B.4). It follows:

$$
\begin{aligned}
F_{0}(t) & =\left(\mu_{X}(t)-\left.\frac{\partial \mu_{X}(t)}{\partial X(t)^{\prime}}\right|_{X(t)=X(t \mid t)} X(t \mid t)\right) \Delta t, \\
F_{1}(t) & =I+\left.\frac{\partial \mu_{X}(t)}{\partial X(t)^{\prime}}\right|_{X(t)=X(t \mid t)} \Delta t, \\
Q(t) & =\sigma_{X}(X(t \mid t)) \sigma_{X}^{\prime}(X(t \mid t)) \Delta t .
\end{aligned}
$$


The term "extended" Kalman filter refers to the fact that $F_{0}(t), F_{1}(t)$ and $Q(t)$ depend on the state variables $X(t)$. For details, see Duffee and Stanton (2002). With $h_{f}(t)=\tilde{h}_{f}(t)=\sigma_{f} r(t)$ and $h_{A}(t)=\tilde{h}_{A}(t)=\sigma_{A} \lambda_{A}(t), F_{0}(t)$ simplifies to:

$$
F_{0}(t)=\left(\begin{array}{c}
\kappa_{f} \theta_{f}-\nu_{f} h_{f}^{2}(t) \\
\kappa_{A} \theta_{A}-\left(\nu_{A} \sqrt{1-\rho^{A^{2}}} h_{A}^{2}(t)+\rho^{A}\left(1+\nu_{f}\right) h_{f}(t) h_{A}(t)\right) \\
-h_{f}^{2}(t) \\
-h_{A}^{2}(t) \\
0 \\
0
\end{array}\right) \Delta t .
$$

The structure for $F_{1}(t)$ also simplifies. Defining $\partial h_{f}(t) \equiv \frac{d}{d t} h_{f}(t)=\sigma_{f}$, and $\partial h_{A}(t) \equiv \frac{d}{d t} h_{A}(t)=\sigma_{A}$, $k_{1}=1, k_{2}=\rho^{A}\left(\frac{\kappa_{f}}{\kappa_{A}}+1\right)$, and $k_{3}=\rho^{A}\left(1-\frac{\kappa_{f}}{\kappa_{A}}\right)$, we have $F_{1}(t)=I+A(t) \Delta t$, where

$$
A(t)=\left(\begin{array}{cccccc}
2 \nu_{f} h_{f}(t) \partial h_{f}(t)-\kappa_{f} & 0 & 1 & 0 & 0 & 0 \\
\rho^{A}\left(1+\nu_{f}\right) \partial h_{f}(t) h_{A}(t) & \nu_{A} \sqrt{1-\rho^{2}} 2 h_{A}(t) \partial h_{A}(t) & 0 & k_{1} & k_{2} & k_{3} \\
& +\rho^{A}\left(1+\nu_{f}\right) h_{f}(t) \partial h_{A}(t)-\kappa_{A} & & & & \\
2 h_{f}(t) \partial h_{f}(t) & 0 & -2 \kappa_{f} & 0 & 0 & 0 \\
0 & 2 h_{A}(t) \partial h_{A}(t) & 0 & -2 \kappa_{A} & 0 & 0 \\
\partial h_{f}(t) h_{A}(t) & h_{f}(t) \partial h_{A}(t) & 0 & 0 & -\kappa_{f}-\kappa_{A} & 0 \\
\partial h_{f}(t) h_{A}(t) & h_{f}(t) \partial h_{A}(t) & 0 & 0 & 0 & -\kappa_{f}
\end{array}\right) .
$$

Similar formulas for $F_{0}(t)$ and $A(t)$ are available if the bounds for $h_{f}(t)$ and $h_{A}(t)$ were binding. 


\section{References}

Ahn, D., R. Dittmar, and R. Gallant, 2002, "Quadratic Term Structure Models: Theory and Evidence," The Review of Financial Studies, 15, 243-288.

Amin, K., and A. Morton, 1994, "Implied Volatility Functions in Arbitrage-Free Term Structure Models," Journal of Financial Economics, 35, 141-180.

Basel II, 2006, "International Convergence of Capital Measurement and Capital Standards: A Revised Framework - Comprehensive Version," Basel Committee on Banking Supervision.

Bedendo, M., L. Cathcart, and L. El-Jahel, 2007, "The Slope of the Term Structure of Credit Spreads: An Empirical Investigation," Journal of Financial Research, 30, 237-257.

Berndt, A., 2007, "Specification Analysis of Reduced-Form Credit Risk Models," Working paper, Carnegie Mellon University.

Bjork, T., and B. Christensen, 1999, "Interest Rate Dynamics and Consistent Forward Rate Curves," Mathematical Finance, 9, 323-348.

Bjork, T., Y. Kabanov, and W. Runggaldier, 1997, "Bond Market Structure in the Presence of Marked Point Processes," Mathematical Finance, 7, 211-223.

Bjork, T., and L. Svensson, 2001, "On the Existence of Finite-Dimensional Realizations for Nonlinear Forward Rate Models," Mathematical Finance, 11, 205-243.

Bliss, R., and P. Ritchken, 1996, "Empirical Tests of Two State Variable Heath Jarrow Morton Models," Journal of Money Credit and Banking, 28, 452-476.

Bohn, J., 1999, "Characterizing Credit Spreads," Working paper, UC Berkeley.

Brandt, M., and D. Chapman, 2002, "Comparing Multifactor Models of the Term Structure," Working paper, Duke University.

Brigo, D., and F. Mercurio, 2001, Interest Rate Models: Theory and Practice. Springer Finance.

Chiarella, C., H. Hung, and T. To, 2009, "The Volatility Structure of the Fixed Income Market Under the HJM Framework: A Nonlinear Filtering Approach," Computational Statistics and Data Analysis, 53, 2061-2074.

Chiarella, C., and O. Kwon, 2002, "Classes of Interest Rate Models under the HJM Framework," Asia-Pacific Financial Markets, 8, 1-22.

Collin-Dufresne, P., R. Goldstein, and J. Helwege, 2003, "Is Credit Event Risk Priced? Modeling Contagion via the Updating of Beliefs," Working paper, Columbia University.

Dai, Q., and K. Singleton, 2000, "Specification Analysis of Affine Term Structure Models," Journal of Finance, 55, 1943-1978.

— 2003, "Term Structure Modeling in Theory and Reality," Review of Financial Studies, 16, 631-678.

Danielsson, J., 2002, "The Emperor has no Clothes: Limits to Risk Modeling," Journal of Banking and Finance, 26, 1273-1296.

— , 2008, "Blame the Models," Forthcoming, Journal of Financial Stability.

Das, S., D. Duffie, N. Kapadia, and L. Saita, 2007, "Common Failings: How Corporate Defaults are Correlated," Journal of Finance, 62, 93-117.

De Jong, F., and P. Santa-Clara, 1999, "The Dynamics of the Forward Interest Rate Curve: A Formulation with State Variables," Journal of Financial and Quantitative Analysis, 34, 131-157.

Duffee, G., 1999, "Estimating the Price of Default Risk," Review of Financial Studies, 12, 197-226.

Duffee, G., and R. Stanton, 2002, "Estimation of Dynamic Term Structure Models," Working paper, UC Berkeley. 
Duffie, D., 1996, Dynamic Asset Pricing Theory. Princeton University Press.

Duffie, D., A. Eckner, G. Horel, and L. Saita, 2008, "Frailty Correlated Default," forthcoming, Journal of Finance.

Duffie, D., and R. Kan, 1996, "A Yield Factor Model of Interest Rates," Mathematical Finance, 6, 379-406.

Duffie, D., J. Pan, and K. Singleton, 2000, "Transform Analysis and Asset Pricing for Affine JumpDiffusions," Econometrica, 68, 1343-1376.

Duffie, D., and K. Singleton, 1999a, "Modeling Term Structures of Defaultable Bonds," Review of Financial Studies, 12, 687-720.

— , 1999b, "Simulating Correlated Defaults," Working paper, Stanford University.

Egloff, D., M. Leippold, and P. Vanini, 2007, "A Simple Model of Credit Contagion," Journal of Banking and Finance, 31, 2475-2492.

Fan, R., A. Gupta, and P. Ritchken, 2003, "Hedging in the Possible Presence of Unspanned Stochastic Volatility: Evidence from Swaption Markets," Journal of Finance, 58, 2219-2248.

— 2007, "On Pricing and Hedging in the Swaption Market: How Many Factors, Really?," Journal of Derivatives, 15, 9-33.

Fons, J., 1994, "Using Default Rates to Model the Term Structure of Credit Risk," Financial Analyst Journal, $50,2532$.

Goncalves, F., and J. Issler, 1996, "Estimating the Term Structure of Volatility and Fixed-Income Derivative Pricing," Journal of Fixed Income, June, 32-39.

Gurkaynak, R., B. Sack, and J. Wright, 2006, "The U.S. Treasury Yield Curve: 1961 to the Present," Paper No. 2006-28, Finance and Economics Discussion Series Divisions of Research \& Statistics and Monetary Affairs Federal Reserve Board, Washington, D.C.

He, J., W. Hu, and L. Lang, 2004, "Credit Spread Curves and Credit Ratings," Working paper, Chinese University of Honk Kong.

Heath, D., R. Jarrow, and A. Morton, 1992, "Bond Pricing and the Term Structure of Interest Rates: A New Methodology for Contingent Claims Valuation," Econometrica, 60, 77-105.

Heath, D., R. Jarrow, A. Morton, and M. Spindel, 1992, "Easier Done Than Said," Risk, 5, 77-80.

Helwege, J., and C. Turner, 1999, "The Slope of the Credit Yield Curve for Speculative Grade Issuers," Journal of Finance, 54, 1869-1884.

Hong, Y., and H. Li, 2005, "Nonparametric Specification Testing for Continuous-Time Models with Application to Spot Interest Rates," Review of Financial Studies, 18, 37-84.

Hull, J., and A. White, 2001, "Valuing Credit Default Swaps II: Modeling Default Correlations," Journal of Derivatives, $8,12-22$.

Inui, K., and M. Kijima, 1998, "A Markovian Framework in Multi-Factor Heath-Jarrow-Morton Models," Journal of Financial and Quantitative Analysis, 33, 423-440.

Jarrow, R., D. Lando, and S. Turnbull, 1997, "A Markov Model for the Term Structure of Credit Risk Spreads," The Review of Financial Studies, 10, 481-523.

Jarrow, R., and F. Yu, 2001, "Counterparty Risk and the Pricing of Defaultable Securities," Journal of Finance, 56, 1765-1799.

Jorion, P., and G. Zhang, 2007, "Good and Bad Credit Contagion: Evidence from Credit Default Swaps," Journal of Financial Economics, 84, 860-883.

Kraft, H., and M. Steffensen, 2007, "Bankruptcy, Counterparty Risk, and Contagion," Review of Finance, $11,210-252$. 
La Chioma, C., and B. Piccoli, 2007, "Heath-Jarrow-Morton Interest Rate Dynamics and Approximately Consistent Forward Rate Curves," Mathematical Finance, 17, 427-447.

Lando, D., and M. Nielsen, 2008, "Correlation in corporate defaults: Contagion or conditional independence?," Working paper, Copenhagen Business School.

Larsen, P., 2007, "Goldman Pays the Price of Being Big," Financial Times, August 13, 2007.

Li, D., 2000, "On Default Correlation: A Copula Function Approach," Journal of Fixed Income, 9, 43-54.

Litterman, R., and J. Scheinkman, 1991, "Common Factors Affecting Bond Returns," Journal of Fixed Income, 1, 54-61.

Longstaff, F., and A. Rajan, 2008, "An Empirical Analysis of the Pricing of Collateralized Debt Obligations," NBER Working Paper No. W12210.

Longstaff, F., P. Santa-Clara, and E. Schwartz, 2001, "The Relative Valuation of Caps and Swaptions: Theory and Empirical Evidence," Journal of Finance, 56, 2067-2109.

Longstaff, F., and E. Schwartz, 1995, "A Simple Approach to Valuing Risky Fixed and Floating Rate Debt," The Journal of Finance, 50:3, 789-819.

Merton, R., 1974, "On the Pricing of Corporate Debt: The Risk Structure of Interest Rates," Journal of Finance, 29, 449-470.

Pan, J., and K. Singleton, 2008, "Default and Recovery Implicit in the Term Structure of Sovereign CDS Spreads," Journal of Finance, 63, 2345-2384.

Piazzesi, M., 2003, "Affine Term Structure Models," Prepared for the Handbook of Financial Econometrics.

Quinn, J., 2008, "Federal Reserve Stress Tests Banks to Avoid Bear Stearns Repeat," Telegraph, August 22, 2008.

Ramaprasad, B., and C. Chiarella, 1995, "Transformations of the Heath-Jarrow-Morton Models to Markovian Systems," European Journal of Finance, 3, 1-26.

Ritchken, P., and L. Sankarasubramanian, 1995, "Volatility Structure of Forward Rates and the Dynamics of the Term Structure," Mathematical Finance, 5, 55-72.

Sarig, O., and A. Warga, 1989, "Some Empirical Estimates of the Risk Structure of Interest Rates," Journal of Finance, 44, 1351-1360.

Schönbucher, P., 2000, "The Term Structure of Defaultable Bond Prices," Review of Derivatives Research, 2, 161-192.

Schönbucher, P., and D. Schubert, 2002, "Copula-Dependent Default Risk in Intensity Models," Working paper, ETH Zurich.

The Economist, 2008, "The Bigger Picture: Enterprise Risk Management in Financial Services Organizations," Economist Intelligence Unit, The Economist.

Vasicek, O., 1977, "An Equilibrium Characterization of the Term Structure," Journal of Financial Economics, $5,177-188$.

Whitehouse, K., 2007, "One "Quant" Sees Shakeout for the Ages - "10,000 Years"," Wall Street Journal, August 11, 2007.

Yu, F., 2005, "Default Correlation in Reduced-Form Models," Journal of Investment Management, 3, 33-42.

Yu, F., 2007, "Correlated Defaults in Intensity-Based Models," Mathematical Finance, 17, 155-173.

Zheng, H., and L. Jiang, 2009, "Basket CDS Pricing with Interacting Intensities," Finance and Stochastics, $13,445-469$. 
Table 1: Estimation Results The top panel shows the extended Kalman filter estimates for a onefactor proportional model of riskless yields. Weekly 1- through 10-year yields are observed with normally distributed measurement errors with variance $\sigma_{\varepsilon_{f}}^{2}$, independent across time and maturities. The value of $\theta_{f}$ is fixed at the sample mean of the 1-year yield. The second panel reports the extended Kalman filter estimates for AMR's credit spread curve. Weekly 1-, 3-, 5-, 7- and 10-year credit spreads are observed with normally distributed measurement errors with variance $\sigma_{\varepsilon_{A}}^{2}$, independent across time and maturities. The value of $\theta_{A}$ is fixed at the sample mean of the 1-year spread. We set $\bar{h}_{f}=\bar{h}_{A}=10^{6}$, but as long as sufficiently large, the precise values of these caps do not alter the estimation results. This analysis is repeated for Lennar. Small-sample Monte Carlo standard error estimates using 100 simulation runs are reported in parentheses. The sample period is February 1, 2005 to May 15, 2009.

\begin{tabular}{ccccccc}
\hline & \multicolumn{6}{c}{ Riskless Rates } \\
& $\theta_{f}$ & $\kappa_{f}$ & $\sigma_{f}$ & $\nu_{f}$ & $100 \sigma_{\varepsilon_{f}}$ & \\
\cline { 2 - 6 } Treasury & 0.034 & 0.106 & 1.300 & -8.753 & 0.287 & \\
& - & $(0.007)$ & $(0.084)$ & $(28.580)$ & $(0.004)$ \\
\hline \multirow{5}{*}{ AMR } & \multicolumn{7}{c}{ Credit Spreads } \\
& $\theta_{A}$ & $\kappa_{A}$ & $\sigma_{A}$ & $\nu_{A}$ & $\rho^{A}$ & $100 \sigma_{\varepsilon_{A}}$ \\
\cline { 2 - 6 } Lennar & 0.118 & 0.041 & 0.422 & 8.750 & -0.120 & 0.456 \\
& - & $(0.003)$ & $(0.015)$ & $(12.153)$ & $(0.059)$ & $(0.005)$ \\
& - & 0.166 & 0.784 & 0.929 & -0.201 & 0.077 \\
& - & $(0.007)$ & $(0.052)$ & $(42.889)$ & $(0.067)$ & $(0.001)$ \\
\hline
\end{tabular}


Table 2: The Importance of Interest Rate-Credit Spread Correlations: Bond Options Simulation results for the prices $c$ (in dollar) of an at-the-money 3-year European call option on a 5-year zerocoupon defaultable bond with a notional amount of $\$ 100$, under different parameter specifications. Standard errors are reported in parentheses. The benchmark set of parameter values is described in Footnote 14. Additional scenario-specific parameters are listed at the end of the table. Our Monte Carlo simulations use 10,000 sample paths with antithetic sampling.

\begin{tabular}{|c|c|c|c|c|c|}
\hline$\rho^{A}$ & $c$ & $\eta_{f}$ & $c$ & $c_{f A}$ & $c$ \\
\hline-0.9 & $\begin{array}{c}2.437 \\
(0.026)\end{array}$ & 0.00 & $\begin{array}{c}3.069 \\
(0.036)\end{array}$ & 0.00 & $\begin{array}{c}3.071 \\
(0.036)\end{array}$ \\
\hline-0.7 & $\begin{array}{c}2.589 \\
(0.029)\end{array}$ & 0.05 & $\begin{array}{c}3.082 \\
(0.036)\end{array}$ & 0.01 & $\begin{array}{c}3.070 \\
(0.036)\end{array}$ \\
\hline-0.5 & $\begin{array}{c}2.736 \\
(0.031)\end{array}$ & 0.10 & $\begin{array}{c}3.085 \\
(0.036)\end{array}$ & 0.02 & $\begin{array}{c}3.073 \\
(0.036)\end{array}$ \\
\hline-0.3 & $\begin{array}{c}2.881 \\
(0.033)\end{array}$ & 0.15 & $\begin{array}{c}3.092 \\
(0.036)\end{array}$ & 0.03 & $\begin{array}{c}3.073 \\
(0.036)\end{array}$ \\
\hline-0.1 & $\begin{array}{c}3.011 \\
(0.035)\end{array}$ & 0.20 & $\begin{array}{c}3.100 \\
(0.036)\end{array}$ & 0.04 & $\begin{array}{c}3.074 \\
(0.036)\end{array}$ \\
\hline 0.0 & $\begin{array}{c}3.069 \\
(0.036)\end{array}$ & 0.25 & $\begin{array}{c}3.112 \\
(0.036)\end{array}$ & 0.05 & $\begin{array}{c}3.075 \\
(0.036)\end{array}$ \\
\hline 0.1 & $\begin{array}{c}3.126 \\
(0.036)\end{array}$ & 0.30 & $\begin{array}{c}3.120 \\
(0.036)\end{array}$ & 0.06 & $\begin{array}{c}3.078 \\
(0.036)\end{array}$ \\
\hline 0.3 & $\begin{array}{c}3.226 \\
(0.037)\end{array}$ & 0.35 & $\begin{array}{c}3.128 \\
(0.037)\end{array}$ & 0.07 & $\begin{array}{c}3.080 \\
(0.036)\end{array}$ \\
\hline 0.5 & $\begin{array}{c}3.339 \\
(0.038)\end{array}$ & 0.40 & $\begin{array}{c}3.138 \\
(0.037)\end{array}$ & 0.08 & $\begin{array}{c}3.082 \\
(0.036)\end{array}$ \\
\hline 0.7 & $\begin{array}{c}3.438 \\
(0.039)\end{array}$ & 0.45 & $\begin{array}{c}3.144 \\
(0.037)\end{array}$ & 0.09 & $\begin{array}{c}3.086 \\
(0.036)\end{array}$ \\
\hline 0.9 & $\begin{array}{c}3.532 \\
(0.040)\end{array}$ & 0.50 & $\begin{array}{c}3.153 \\
(0.037)\end{array}$ & 0.10 & $\begin{array}{c}3.090 \\
(0.036)\end{array}$ \\
\hline \multicolumn{6}{|c|}{ Parameter Specifications } \\
\hline \multicolumn{2}{|c|}{$\eta_{f}=0$} & \multicolumn{2}{|c|}{$c_{f A}=0.025$} & \multicolumn{2}{|c|}{$\eta_{f}=0.01$} \\
\hline
\end{tabular}


Table 3: The Importance of Interest Rate-Credit Spread Correlations: CCDS Simulation results for at-market rates $C$ (in basis points) of a 5-year CCDS contract on a 5-year floating-for-fixed interest-rate swap, under different parameter specifications. Standard errors are reported in parentheses. The benchmark set of parameter values is described in Footnote 14, with A replaced by B. Additional scenario-specific parameters are listed at the end of the table. Our Monte Carlo simulations use 10,000 sample paths with antithetic sampling.

\begin{tabular}{cccccc}
\hline$\rho^{B}$ & $C$ & $\eta_{f}$ & $C$ & $c_{f B}$ & $C$ \\
\hline-0.9 & 23.771 & 0.000 & 17.957 & 0.000 & 17.922 \\
& $(0.616)$ & & $(0.523)$ & & $(0.522)$ \\
-0.7 & 22.433 & 0.050 & 17.957 & 0.010 & 17.956 \\
& $(0.594)$ & & $(0.525)$ & & $(0.522)$ \\
-0.5 & 21.198 & 0.100 & 18.045 & 0.020 & 17.926 \\
& $(0.575)$ & & $(0.528)$ & & $(0.522)$ \\
-0.3 & 19.857 & 0.150 & 18.223 & 0.030 & 17.955 \\
& $(0.555)$ & & $(0.531)$ & & $(0.523)$ \\
-0.1 & 18.584 & 0.200 & 18.209 & 0.040 & 17.968 \\
& $(0.534)$ & & $(0.531)$ & & $(0.523)$ \\
0.0 & 17.957 & 0.250 & 18.296 & 0.050 & 17.985 \\
& $(0.523)$ & & $(0.534)$ & & $(0.523)$ \\
0.1 & 17.395 & 0.300 & 18.411 & 0.060 & 17.994 \\
& $(0.513)$ & & $(0.536)$ & & $(0.523)$ \\
0.3 & 16.452 & 0.350 & 18.505 & 0.070 & 18.027 \\
& $(0.498)$ & & $(0.538)$ & & $(0.524)$ \\
0.5 & 15.038 & 0.400 & 18.578 & 0.080 & 18.055 \\
& $(0.472)$ & & $(0.540)$ & & $(0.524)$ \\
0.7 & 13.713 & 0.450 & 18.678 & 0.090 & 18.032 \\
& $(0.448)$ & & $(0.543)$ & & $(0.524)$ \\
0.9 & 12.602 & 0.500 & 18.933 & 0.100 & 18.061 \\
& $(0.426)$ & & $(0.548)$ \\
& & Parameter Specifications & & $(0.525)$ \\
& $\eta_{f}=0$ & \multicolumn{2}{c}{$c_{f B}=0.025$} & & \\
\hline
\end{tabular}


Table 4: The Importance of the Initial Credit Spread Curve Distribution Simulated tranche spreads (in basis points) of the 5-year CDX.NA.HY index, for different distributions of initial credit spread curves across the firms in the index. The benchmark set of parameter values is described in Footnote 18. We set $\eta_{f}=0$, and there are no feedback effects from defaults of primary firms.

\begin{tabular}{lrrc}
\hline Distribution of initial credit spread curves & \multicolumn{3}{c}{ Tranche spreads } \\
& $10-15$ & $15-25$ & $25-35$ \\
\hline$\lambda(0, t)=0.05$ & 3451 & 1450 & 111 \\
$\lambda(0,0) \sim$ Uniform $(0.025,0.075)$ and $\lambda(0, t)=\lambda(0,0)$ & 3523 & 1528 & 128 \\
$\lambda(0,0) \sim$ Uniform $(0,0.1)$ and $\lambda(0, t)=\lambda(0,0)$ & 3528 & 1528 & 121 \\
$\lambda(0, t)$ increases linearly from $\lambda(0, t)=0.0125$ to $\lambda(0, t)=0.0875$ & 2698 & 1340 & 107 \\
$\lambda(0, t)$ increases linearly from $\lambda(0, t)=0.025$ to $\lambda(0, t)=0.075$ & 2883 & 1366 & 108 \\
$\lambda(0, t)$ increases linearly from $\lambda(0, t)=0.0375$ to $\lambda(0, t)=0.0625$ & 3130 & 1405 & 110 \\
$\lambda(0, t)=0.05$ & 3451 & 1450 & 111 \\
$\lambda(0, t)$ decreases linearly from $\lambda(0, t)=0.0625$ to $\lambda(0, t)=0.0375$ & 3857 & 1514 & 115 \\
$\lambda(0, t)$ decreases linearly from $\lambda(0, t)=0.075$ to $\lambda(0, t)=0.025$ & 4345 & 1601 & 120 \\
$\lambda(0, t)$ decreases linearly from $\lambda(0, t)=0.0875$ to $\lambda(0, t)=0.0125$ & 4947 & 1772 & 141 \\
\hline
\end{tabular}




\section{Panel A: Riskless Yield Curves}

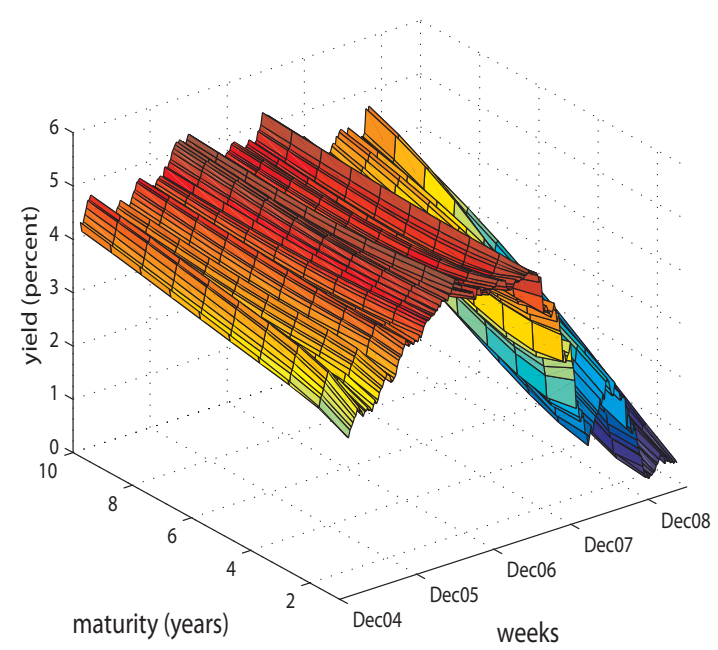

Panel B: Credit Spread Curves
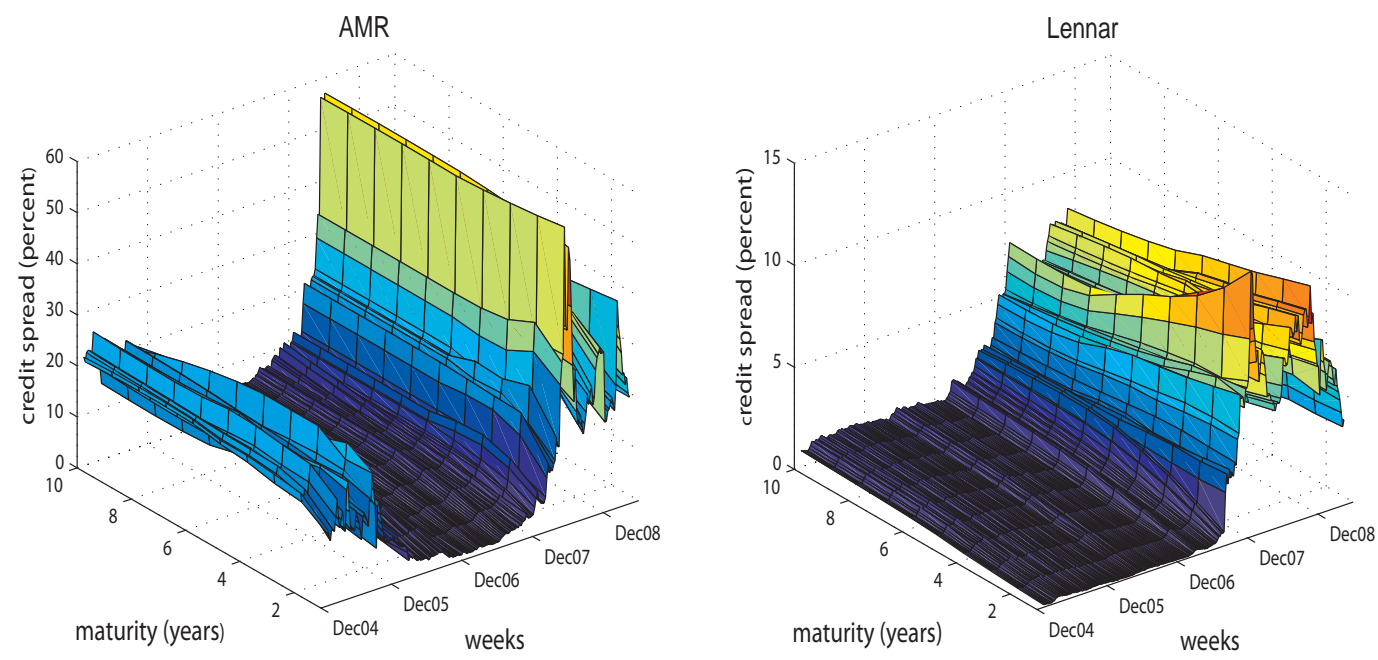

Figure 1: Riskless Yield Curves and Credit Spreads The top panel shows the weekly time series of riskless yields to maturity from February 1, 2005 to May 15, 2009. The bottom panel shows the weekly time series of credit spreads for AMR (left) and Lennar (right) over the same period. This data is provided by Gurkaynak, Sack and Wright (riskless yields) and CMA (credit spreads). 

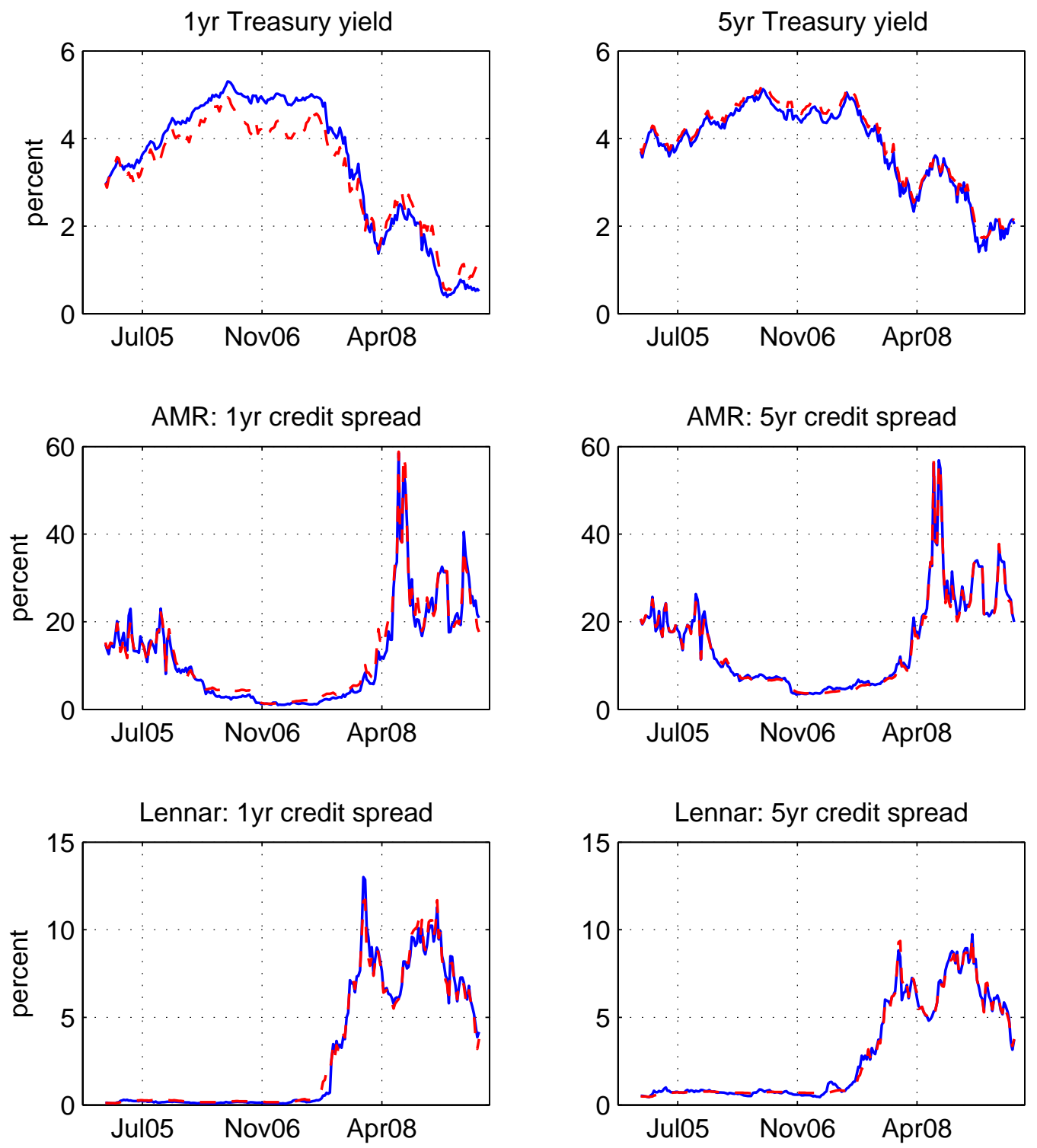

Figure 2: Estimation Results This plot shows the observed (solid line) and fitted (dashed line) time series of 1- and 5-year riskless yields and of 1- and 5-year credit spreads for AMR and Lennar. The sample period is February 1, 2005 to May 15, 2009. 


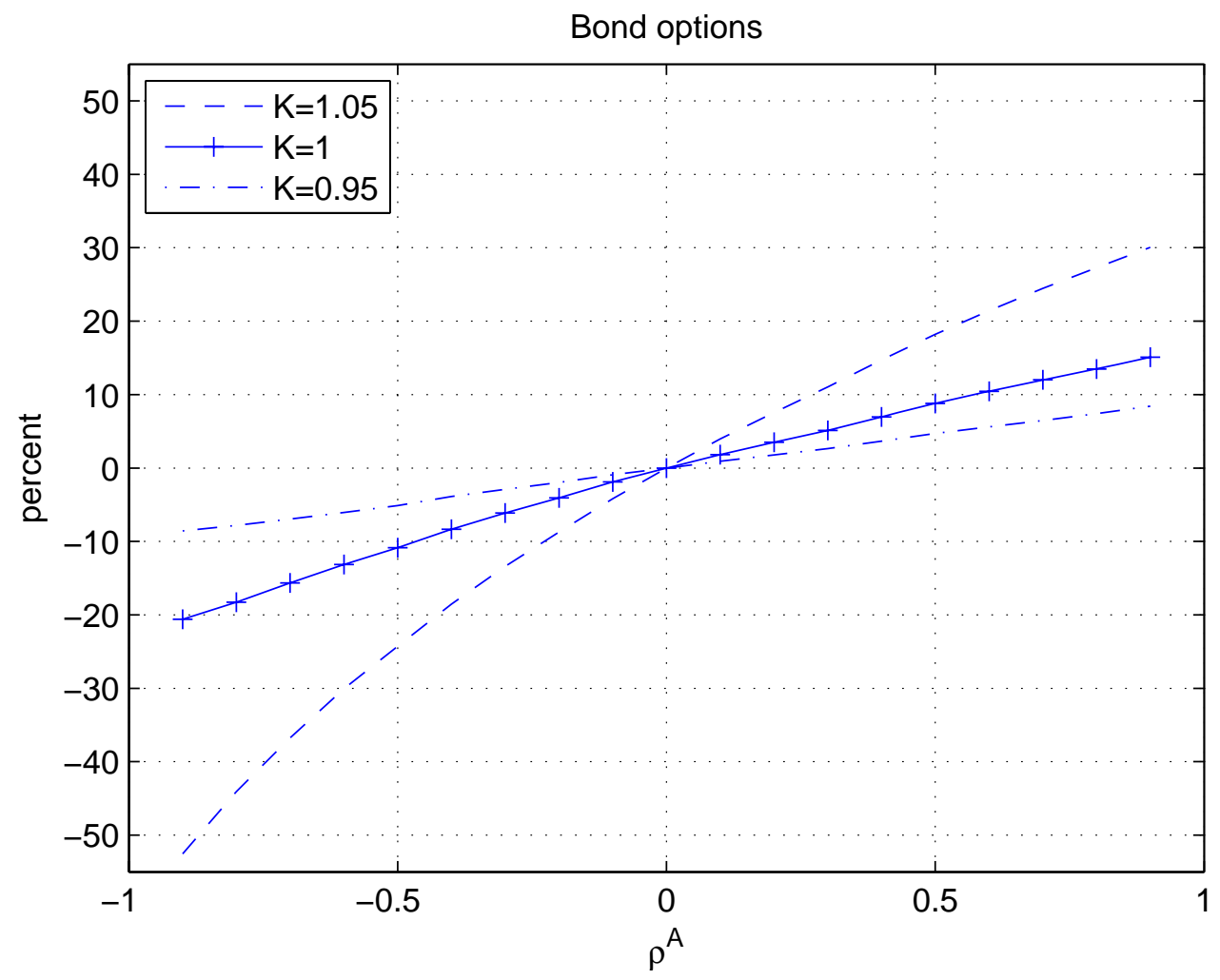

Figure 3: The Importance of Interest Rate-Credit Spread Correlations Percentage changes in the prices of a 3-year European call option on a 5 -year zero-coupon defaultable bond for varying $\rho^{A}$, relative to the value at $\rho^{A}=0$. The strike price of the bond options is equal to $K$ times the forward price, for different $K$. The benchmark set of parameter values is described in Footnote 14. We set $\eta_{f}=0$. 

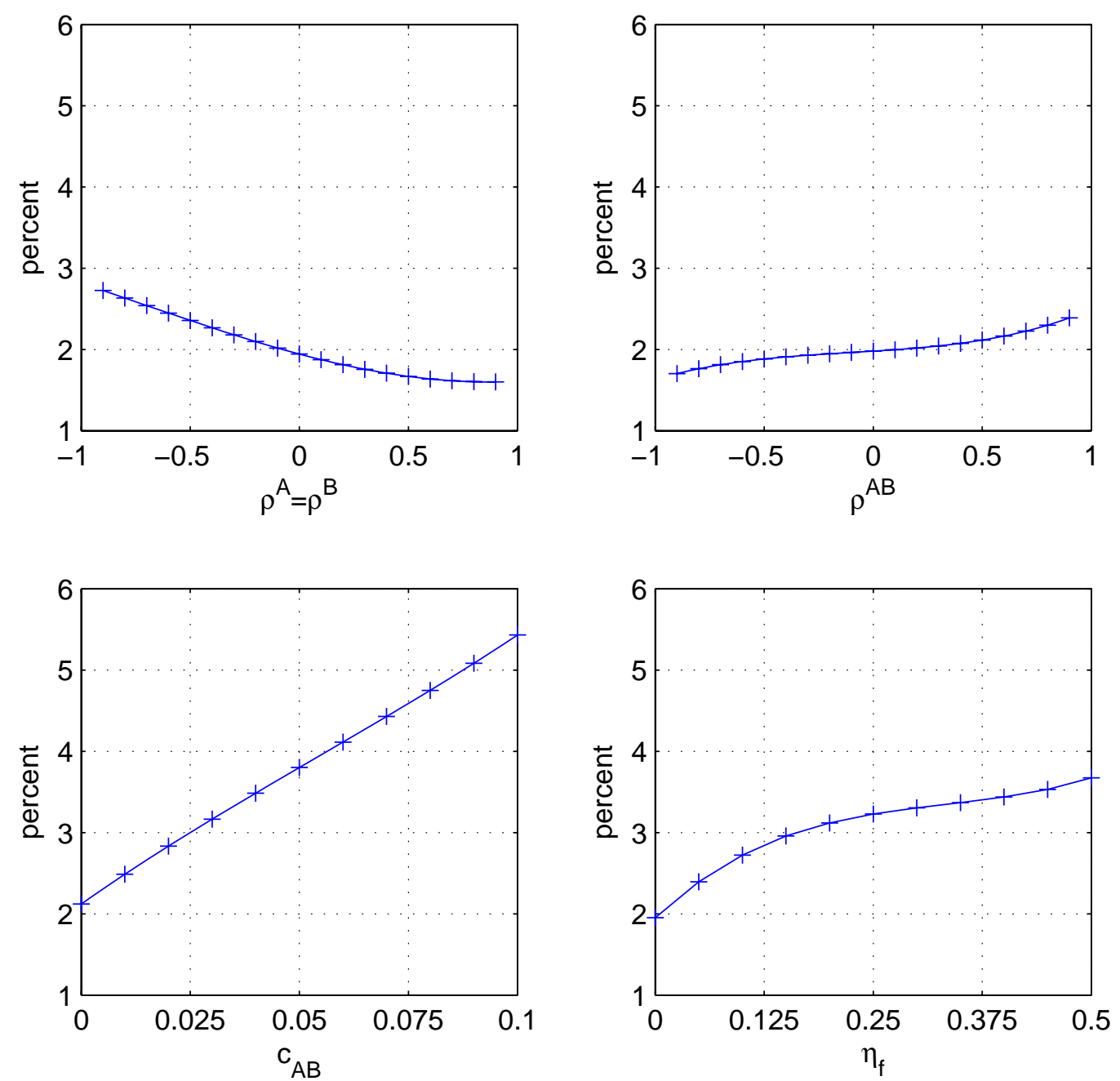

Figure 4: The Importance of Default Contagion: Counterparty Risk in Insurance Contracts Reduction in at-market CCDS rates due to the default risk of the protection seller, expressed as a fraction of the at-market CCDS rates when the default risk of the protection seller is ignored, for varying $\rho^{A}=\rho^{B}, \rho^{A B}, c_{A B}$ and $\eta_{f}$. The benchmark set of parameter values is described in Footnote 18, with the number of sample paths increased to 50,000 . We set $\lambda_{A}(0, t)=0.01$ and $\lambda_{B}(0, t)=0.05$, and, when $\eta_{f}$ is away from zero, $c_{f A}=0.005$ and $c_{f B}=0.025$. 

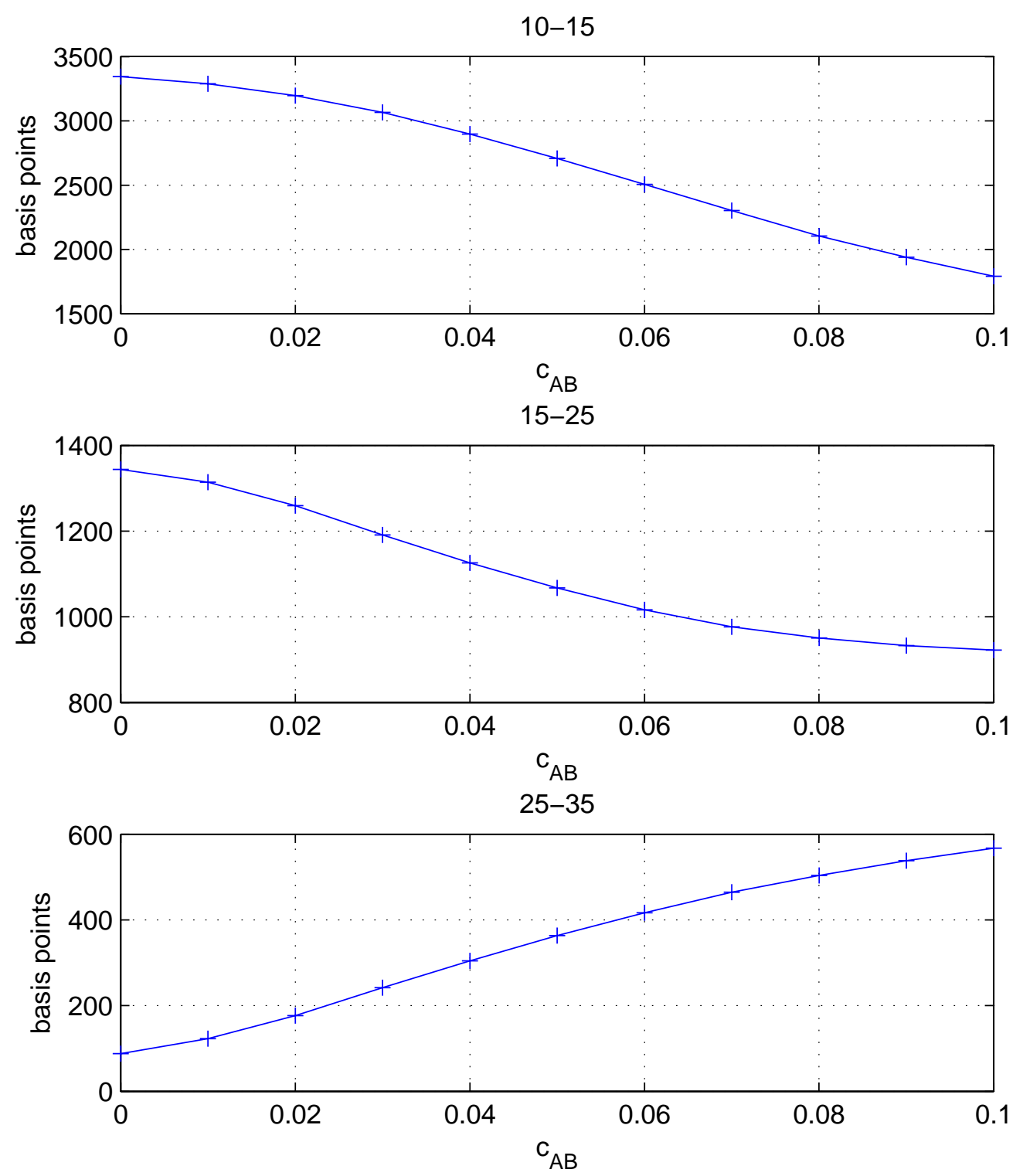

Figure 5: The Importance of Default Contagion: CDS Index Tranches Simulated tranche spreads of the 5-year CDX.NA.HY index, as a function of $c_{A B}$. The benchmark set of parameter values is described in Footnote 18. There are 5 primary firms and 95 secondary firms. We set $\lambda_{A}(0, t)=0.025$ and $\lambda_{B}(0, t)=0.05$. 

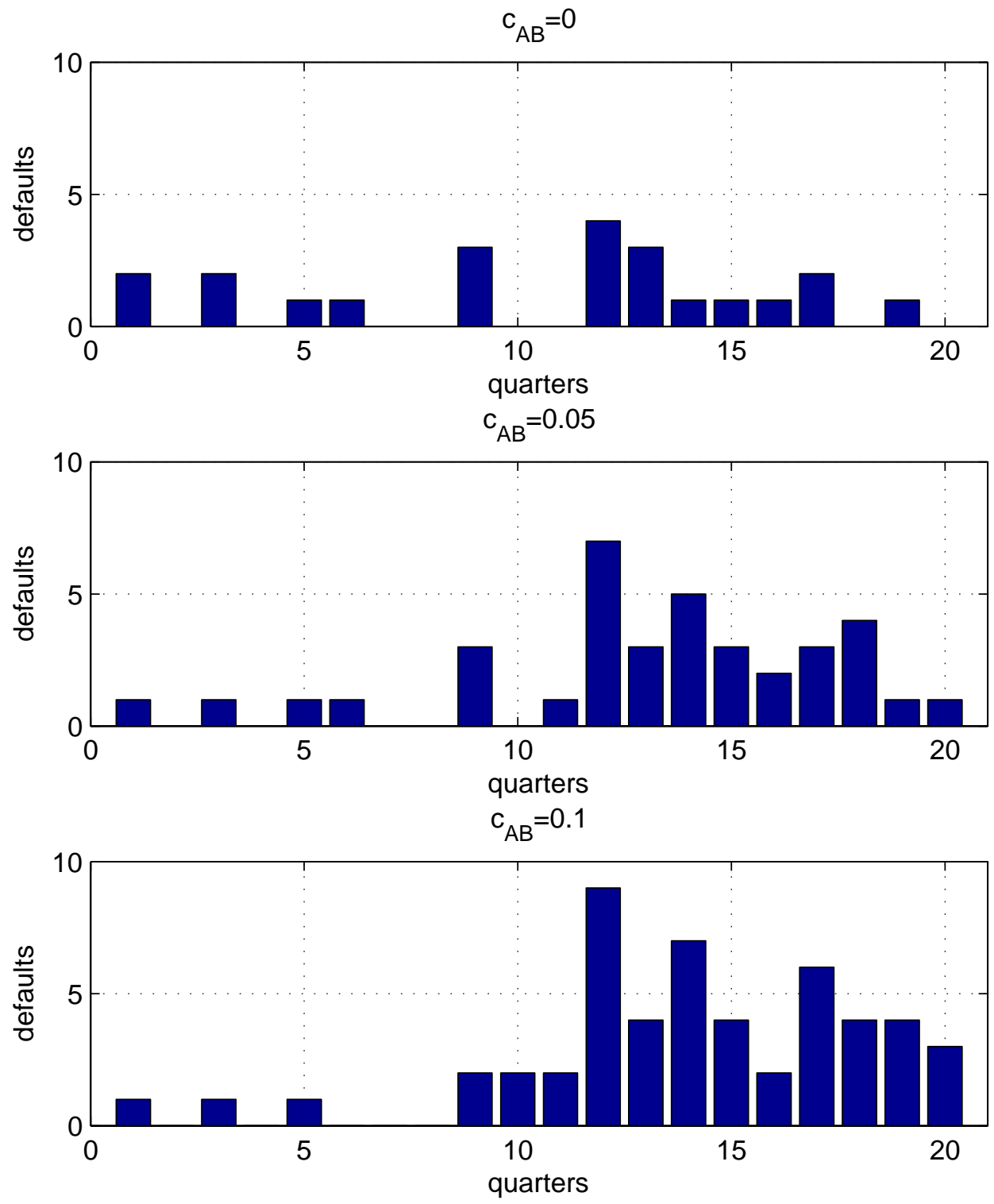

Figure 6: Generating Default Clustering Sample path of the number of defaults across time. The benchmark set of parameter values is described in Footnote 18. There are 5 primary firms and 95 secondary firms. We set $\lambda_{A}(0, t)=0.025$ and $\lambda_{B}(0, t)=0.05$. 